\title{
Survey of the Theories for Analogue of Wiener Measure Space
}

\author{
Kun Sik RYU ${ }^{1, *}$, Man Kyu IM² and Ki Seong $\mathrm{CHOI}^{3}$ \\ ${ }^{1}$ Department of Mathematics Education, Han Nam University, Daejon 306-791, Korea \\ ${ }^{2}$ Department of Mathematics, Han Nam University, Daejeon 306-791, Korea \\ ${ }^{3}$ Department of Information Security, Konyang University, Nonsan 320-711, Korea
}

Received January 28, 2009; final version accepted June 3, 2009

\begin{abstract}
We consider the analogue of Wiener measure on the space of all real continuous functions, and then establish the measure-valued measure and the operator-valued measure on our space and we investigate their important properties, for example, the integration formula, the rotation theorem, the Bartle integral, the Bochner integral and the Dobrakov integral, the simple formula for conditional expectation, the measure-valued Feynman-Kac formula and Volterra integral equation and integral transform.
\end{abstract}

KEYWORDS: analogue of Wiener measure, Bartle integral, Bochner integral, Dobrakov integral, conditional expectation, measure-valued Feynman-Kac formula, integral transform

\section{Historical Background}

Robert Brown, the British botanist, observed the motion of small particles while viewing pollen suspended in water through a microscope in 1827. He hypothesized that this motion was either due to a particular phenomenon of living matter or the power source of life. So, the novelty was investigated extensively and deeply by many scientists of the time, among whom were Cantoni, Oehl, Delsaux, Exner and Guoy. The results of these efforts showed that Brown's original hypotheses were wrong and this motion is very irregular and rapid. This motion is called the Brownian motion.

Albert Einstein, in 1905, suggested a probabilistic approach to Brownian motion in his paper that is associated with the special theory of relativity. His suggestion is that if at time $a$, a particle is at the point 0 in the space, having diffusion constant $D$ and this particle moves like the Brownian motion then the probability $P_{t}(E)$ of finding in a region $E$ after time $t$, is

$$
P_{t}(E)=(4 \pi t D)^{-\frac{3}{2}} \int_{E} \exp \left\{-\frac{\|u\|^{\frac{1}{2}}}{4 t D}\right\} d u .
$$

From Einstein's suggestion, Nobert Wiener established the existence theorem of a probability measure $m_{w}$ on $C_{0}[a, b]$ which satisfies almost all previous results of researcher for Brownian motion, here, $C_{0}[a, b]$ is the space of all realvalued continuous functions on a closed interval $[a, b]$ that vanish at the origin $a$, the so-called Wiener space in 1923 [38]. Since then, the theories of Wiener measure space arose the many new questions in mathematics and theories, so many mathematicians and many physicists were investigate it extensively and deeply. And many new research areas in mathematics and mathematical physics accrue from it. But, the theory of Wiener measure space is a theory of single particle, merely. The authors wanted to make a measure theory on many particles moving along the law of Brownian motion. For simplicity, we ignored the collision between particles. From these concepts, we established the definition and some theories of analogue of Wiener measure space in 2002 [36].

In this note, we introduce the definitions and properties for analogue of Wiener measure, measure-valued measure on analogue of Wiener space and operator-valued measure on analogue of Wiener space. We investigate the simple formula for conditional expectation, the Bartle integral the Bochner integral and the Dobrakov integral on our measure space.

\section{Preliminaries}

In this section, we present some notation, definitions and well-known facts which are needed to understand the subsequent sections.

(A) Let $\mathbb{R}$ be the real number system and let $\mathbb{C}$ be the complex number system. For a natural number $n$, let $\mathbb{R}^{n}$ be the $n$-times product space of $\mathbb{R}$. Let $\mathcal{B}(\mathbb{R})$ be the set of all Borel measurable subsets of $\mathbb{R}$ and let $m_{L}$ be the Lebesgue measure on the measurable space $(\mathbb{R}, \mathscr{B}(\mathbb{R}))$. Let $\alpha_{1}=1, \alpha_{2}=-1, \alpha_{3}=i$ and $\alpha_{4}=-i$. 
(B) For a positive real number $a, b$, let $C[a, b]$ be the space of all real-valued continuous functions on a closed bounded interval $[a, b]$ with the supremum norm $\|\cdot\|_{\infty}$. By the Stone-Weierstrass theorem,

$\left(C[a, b],\|\cdot\|_{\infty}\right)$ is a real separable Banach space.

Let $\mathcal{M}(\mathbb{R})$ be the space of all finite complex-valued countably additive measure on $(\mathbb{R}, \mathscr{B}(\mathbb{R}))$. For $p$ in $\mathbb{R}$, let $\delta_{p}$ be the Dirac measure concentrated at $p$ with total mass one. For $\mu$ in $\mathcal{M}(\mathbb{R})$ and for $E$ in $\mathscr{B}(\mathbb{R})$, the total variation $|\mu|(E)$ on $E$ is defined by

$$
|\mu|(E)=\sup \sum_{i=1}^{n}\left|\mu\left(E_{i}\right)\right|
$$

where the supremum is taken over all finite sequences $\left\langle E_{i}\right\rangle$ of disjoint sets in $\mathcal{B}(\mathbb{R})$. Then $|\mu|$ is in $\mathcal{M}(\mathbb{R})$ and, by the Jordan decomposition theorem $[14]\left((19.13)\right.$ Theorem, p 307), there are unique non-negative measures $\mu_{j}(j=1,2,3,4)$ in $\mathcal{M}(\mathbb{R})$ such that

$$
\mu=\sum_{j=1}^{4} \alpha_{j} \mu_{j}
$$

By Theorem 4.1.7 in $[9],(\mathcal{M}(\mathbb{R}),|\cdot|(\mathbb{R}))$ is a complex Banach space.

Let $\mathcal{R} \mathcal{M}(\mathbb{R})$ be the space of all finite complex-valued measures $\mu$ on $(\mathbb{R}, \mathcal{B}(\mathbb{R}))$ which are absolutely continuous with respect to $m_{L}$, that is, the Radon-Nikodim derivative $\frac{d|\mu|}{d m_{L}}$ exists.

(C) Let $(X, \mathcal{B}, \mu)$ be a measure space. For a positive real number $p$, let $\mathcal{L}^{p}(X, \mu)$ be the space of complex-valued $\mu$-measurable functions $f$ on $X$ such that $|f|^{p}$ is $|\mu|$-integrable. Let $\mathscr{L}^{\infty}(X, \mu)$ be the space of complex-valued $\mu$-measurable functions $f$ on $X$ which are $|\mu|$-essentially bounded. The elements of $\mathcal{L}^{p}(X, \mu)$ and $\mathcal{L}^{\infty}(X, \mu)$ are equivalence classes of functions in $\mathcal{L}^{p}(X, \mu)$ and $\mathcal{L}^{\infty}(X, \mu)$, respectively, with the equivalence relation being defined by $|\mu|$-a.e. Since $\mathcal{R} \mathcal{M}(\mathbb{R})$ is isomorphic to $L^{1}\left(\mathbb{R}, m_{L}\right), \mathcal{R} \mathcal{M}(\mathbb{R})$ is a Banach space and the dual space $\mathcal{R} \mathcal{M}(\mathbb{R})^{*}$ of $\mathcal{R} \mathcal{M}(\mathbb{R})$ is isomorphic to $L^{\infty}\left(\mathbb{R}, m_{L}\right)$. For $x^{*}$ in $\mathcal{R} \mathcal{M}(\mathbb{R})^{*}$, there is a function $\theta$ in $L^{\infty}\left(\mathbb{R}, m_{L}\right)$ such that $x^{*}(\mu)=$ $\int_{\mathbb{R}} \theta(s) d \mu(s)$ for $\mu$ in $\mathcal{R} \mathcal{M}(\mathbb{R})$.

Let $\mathbb{B}$ be a complex Banach space and let $\mathbb{B}^{*}$ be the dual space of $\mathbb{B}$. For a $\mathbb{B}$-valued countably additive measure $v$ on $(X, \mathcal{B})$ and for $E$ in $\mathscr{B}$, the semivariation $\|v\|(E)$ of $v$ on $E$ is given by

$$
\|\nu\|(E)=\sup \left\{\left|x^{*} \nu\right|(E) \mid x^{*} \text { is in } \mathbb{B}^{*} \text { and }\left\|x^{*}\right\|_{\mathbb{B}^{*}} \leq 1\right\}
$$

where $\left|x^{*} \nu\right|(E)$ is the total variation on $E$ of the complex-valued measure $x^{*} v$.

(D) Let $\mathbb{B}$ be a complex Banach space and let $(X, \mathcal{B}, \mu)$ be a complex measure space. A function $f: X \rightarrow \mathbb{B}$ is called $\mu$-measurable if there exists a sequence $\left\langle f_{n}\right\rangle$ of $\mathbb{B}$-valued simple functions with

$$
\lim _{n \rightarrow \infty}\left\|f_{n}-f\right\|_{\mathbb{B}}=0, \quad|\mu| \text {-a.e. }
$$

A function $f$ is called $\mu$-weakly measurable if $x^{*} f$ is $\mu$-measurable for each $x^{*}$ in $\mathbb{B}^{*}$, the dual space of $\mathbb{B}$. By the Pettis's measurability theorem [10],

$$
\begin{aligned}
& f \text { is } \mu \text {-measurable if and only if } f \text { is }|\mu| \text {-essentially } \\
& \text { separably valued and } f \text { is } \mu \text {-weakly measurable. }
\end{aligned}
$$

We say that $f$ is $\mu$-Bochner integrable if there exists a sequence $\left\langle f_{n}\right\rangle$ of $\mathbb{B}$-valued simple functions such that $\left\langle f_{n}\right\rangle$ converges to $f$ in the norm sense in $\mathbb{B}$ for $|\mu|$-a.e. and $\lim _{n \rightarrow \infty} \int_{X}\left\|f(t)-f_{n}(t)\right\|_{\mathbb{B}} d|\mu|(t)=0$. In this case, $(B o)-\int_{X} f(t) d \mu(t)$ is defined by

$$
(B o)-\int_{X} f(t) d \mu(t)=\lim _{n \rightarrow \infty} \int_{X} f_{n}(t) d \mu(t)
$$

where the limit means the limit in the norm sense. By [10][4, Theorem 2, p 45],

$$
f \text { is } \mu \text {-Bochner integrable if and only if } \int_{X}\|f(t)\|_{\mathbb{B}} d|\mu|(t) \text { is finite. }
$$

By Corollary 2 in [42],

if $U$ is a bounded linear operator on $\mathbb{B}$ into a Banach

$\mathbb{B}_{1}$ and $f$ is a $\mathbb{B}$-valued $\mu$-Bochner integrable function,

then $U f$ is a $\mathbb{B}_{1}$-valued $\mu$-Bochner integrable function, and

$$
(B o)-\int_{X}(U f)(t) d \mu(t)=U\left((B o)-\int_{X} f(t) d \mu(t)\right) .
$$

Theorem 2.1. Let $(X, \mathcal{B}, \mu)$ be a complex measure space and let $f: X \rightarrow \mathcal{M}(\mathbb{R})$ be a $\mu$-Bochner integrable function. Then for $E$ in $\mathscr{B}(\mathbb{R}),[f(t)](E)$ is a complex-valued $\mu$-integrable function of $t$ and 


$$
\left[(B o)-\int_{X} f(t) d \mu(t)\right](E)=\int_{X}[f(t)](E) d \mu(t) .
$$

Remark 2.2. Consider a function $H$ on $[0,1] \times[0,1]$ defined by $H(x, y)=\chi_{[0, x]}(y)$. Then $H$ is $m_{L} \times m_{L}$-integrable on $[0,1] \times[0,1]$, so by the Fubini theorem, $H(x, y)$ is an $m_{L}$-integrable function of $x$ for all $y$ and $H(x, \cdot)$ is in $L^{\infty}\left([0,1], m_{L}\right)$ for all $x$ in $[0,1]$. But $H(x, \cdot)$ has no essentially separable range, so $H(x, \cdot)$ is not $m_{L^{-}}$Bochner integrable. Hence, in generally, the equality 2.10 is not true in the theory of Bochner integral.

(E) Let $\mathbb{B}$ be a complex Banach space and let $(Y, \mathcal{C}, v)$ be a $\mathbb{B}$-valued measure space. Let $g$ be a complex-valued $\|v\|$ measurable function on $Y$, that is, there exists a sequence $\left\langle g_{n}\right\rangle$ of complex-valued simple functions with $\lim _{n \rightarrow \infty}\left|g_{n}-g\right|=0$, $\|v\|$-a.e. We say that $g$ is $v$-Bartle integrable if there exists a sequence $\left\langle g_{n}\right\rangle$ of simple functions such that $\left\langle g_{n}\right\rangle$ converges to $g,\|v\|$-a.e. and the sequence $\left\langle\int_{Y} g_{n}(s) d \nu(s)\right\rangle$ is Cauchy in the norm sense. In this case, $(B a)-\int_{Y} g(s) d \nu(s)$ is defined by

$$
(B a)-\int_{Y} g(s) d \nu(s)=\lim _{n \rightarrow \infty} \int_{Y} g_{n}(s) d \nu(s),
$$

where the limit means the limit in the norm sense. By Theorem 8 in [12],

if $f$ is a $v$-measurable function which is $\|v\|$-essentially bounded,

then $f$ is $v$-Bartle integrable and

$$
\left\|(B a)-\int_{Y} f(s) d v(s)\right\|_{\mathbb{B}} \leq(\|v\|-\text { ess. } \sup |f(s)|)\|v\|(Y) .
$$

By Theorem 2.4 in [24],

$g$ is $\nu$-Bartle integrable if and only if for each $x^{*} \in \mathbb{B}^{*}$,

$g$ is $x^{*} v$-integrable and for each $E \in \mathcal{C}$, there is an element

$(B a)-\int_{E} g(s) d \nu(s)$ in $\mathbb{B}$ such that

$$
x^{*}\left[(B a)-\int_{E} g(s) d v(s)\right]=\int_{E} g(s) d x^{*} v(s) \text { for } x^{*} \in \mathbb{B}^{*} \text {. }
$$

By Theorem 8 in [12],

if $U$ is a bounded linear operator from $\mathbb{B}$ into a Banach space $\mathbb{B}_{1}$ and $g$ is $v$-Bartle integrable, then $g$ is $U v$-Bartle integrable.

In this case,

$$
U\left[(B a)-\int_{Y} g(s) d \nu(s)\right]=(B a)-\int_{Y} g(s) d U \nu(s) .
$$

By Theorem 10 in [12],

if $\left\langle f_{n}\right\rangle$ is a sequence of $v$-Bartle integrable functions

which converges $\|v\|$-a.e. to $f$ and if $g$ is a $v$-Bartle integrable function such that $\left|f_{n}(s)\right| \leq g(s)\|v\|$-a.e. $s$ for all natural numbers $n$ then $f$ is $v$-Bartle integrable and for $E$ in $\mathcal{C},(B a)-\int_{E} f(s) d v(s)=\lim _{n \rightarrow \infty}(B a)-\int_{E} f_{n}(s) d \nu(s)$.

(F) Let $\mathbb{B}$ be a complex Banach space. Let $(X, \mathcal{B})$ and $(Y, \mathcal{C})$ be two measurable spaces and let $\mathscr{B} \otimes \mathcal{C}$ be the $\sigma$-algebra of sets in the space $X \times Y$ generated by the family of rectangles $E \times F$ for all $E$ in $\mathscr{B}$ and $F$ in $\mathcal{C}$. Let $\mu$ be a complex-valued measure on $(X, \mathcal{B})$ and let $v$ be a $\mathbb{B}$-valued measure on $(Y, \mathcal{C})$. For $G$ in $\mathcal{B} \otimes \mathcal{C}$, we let

$$
(\mu \times v)(G)=(B a)-\int_{Y}\left[\int_{X} \chi_{G}(u, v) d \mu(u)\right] d \nu(v) .
$$

By Proposition 2 in [18], using the dominated convergence theorem in [19], Kluvanek proved that $\mu \times v$ is a $\mathbb{B}$-valued measure on $\mathcal{B} \otimes \mathcal{C}$ and for $G$ in $\mathcal{B} \otimes \mathcal{C}$,

$$
\begin{aligned}
(\mu \times v)(G) & =(B a)-\int_{Y}\left[\int_{X} \chi_{G}(u, v) d \mu(u)\right] d \nu(v) \\
& =(B o)-\int_{X}\left[(B a)-\int_{Y} \chi_{G}(u, v) d \nu(v)\right] d \mu(u)
\end{aligned}
$$


holds. Moreover, in Proposition 3 in [18], he showed that

$$
x^{*}(\mu \times v)=\mu \times\left(x^{*} v\right)
$$

for all $x^{*}$ in $\mathbb{B}^{*}$.

When both measures $\mu$ and $v$ are complex-valued, a sufficient condition for validity of the Fubini theorem is the integrability of the function with respect to $\mu \times v$. But, if $v$ is a vector measure then the integrability of the function with respect to $\mu \times v$ is no longer a sufficient condition for the validity of the Fubini theorem. Indeed, we can find a counter example for this fact in [18].

Theorem 2.3. Let $\mathbb{B}$ be a separable complex Banach space, $(X, \mathcal{B}, \mu)$ be a complex-valued measure space and let $(Y, \mathcal{C}, v)$ be a $\mathbb{B}$-valued measure space. Let $f: X \times Y \rightarrow \mathbb{C}$ be $\mathbb{B} \otimes \mathcal{C}$-measurable and $\mu \times v$-Bartle integrable. Then

$$
\begin{gathered}
\text { for }\|v\| \text {-a.e. } v, \quad f(u, v) \text { is a } \mu \text {-integrable function of } u \text {, } \\
\int_{X} f(u, v) d \mu(u) \text { is } v \text {-Bartle integrable and } \\
(B a)-\int_{X \times Y} f(u, v) d \mu \times v(u, v)=(B a)-\int_{Y}\left[\int_{X} f(u, v) d \mu(u)\right] d v(v) .
\end{gathered}
$$

Moreover, if for $|\mu|$-a.e. $u, f(u, v)$ is a v-Bartle integrable function of $v$ and $(B a)-\int_{Y} f(u, v) d \nu(v)$ is $\mu$-Bochner integrable then

$$
\begin{aligned}
(B a) & -\int_{X \times Y} f(u, v) d \mu \times v(u, v) \\
& =(B o)-\int_{X}\left[(B a)-\int_{Y} f(u, v) d \nu(v)\right] d \mu(u) \\
& =(B a)-\int_{Y}\left[\int_{X} f(u, v) d \mu(u)\right] d \nu(v) .
\end{aligned}
$$

(G) Let $\mathbb{X}$ and $\mathbb{Y}$ be (real or complex) Banach spaces and denote by $L(\mathbb{X}, \mathbb{Y})$ the Banach space of all bounded linear operator from $\mathbb{X}$ to $\mathbb{Y}$. Let $T$ be a non-empty set and $\mathcal{B}$ a $\sigma$-algebra of subsets of $T$. We say that a set function $m: \mathscr{B} \rightarrow L(\mathbb{X}, \mathbb{Y})$ is an operator-valued countably additive in the strong operator topology if for every $\mathbf{x}$ in $\mathbb{X}$ the set function $E \rightarrow m(E) \mathbf{x}, E \in \mathscr{B}$, is a countable additive vector measure. We define a non negative set function $\hat{m}$, which is called the semivariation of the measure $m$ by equality

$$
\begin{aligned}
& \hat{m}(E)=\sup \left\{\left|\sum_{i=1}^{n} m\left(E \cap E_{i}\right) \mathbf{x}_{i}\right| \mid E_{i} \text { is in } \mathcal{B}, \mathbf{x}_{i} \text { is in } \mathbb{X}\right. \\
& \text { with } \left.\left|\mathbf{x}_{i}\right| \leq 1 \text { for } i=1,2, \ldots, n \text { and } E_{i} \cap E_{j} \emptyset \text { for } i \neq j\right\} .
\end{aligned}
$$

We say that $E$ is an integrable subset in $\mathscr{B}$ if the semivariation $\hat{m}(E)$ of $E$ is finite. Let $\mathcal{K}$ be the set of all integrable subsets of $T$. From [11], we have following theorem.

Theorem 2.4 (*-Theorem). Let $\mathbb{Y}$ contains no subspace isomorphic to the space $c_{0}$, for example let $\mathbb{Y}$ be a weakly complete Banach space. Then the semivariation $\hat{m}$ is continuous on $\mathcal{K}$, that is, if $\left\langle E_{n}\right\rangle$ is a sequence of decreasing subsets in $\mathcal{K}$ with $\lim _{n \rightarrow \infty} E_{n}=\emptyset$ then $\lim _{n \rightarrow \infty} \hat{m}\left(E_{n}\right)=0$.

A $\mathcal{K}$-simple function on $T$ with values in $\mathbb{X}$ is called the simple integrable function. For any simple integrable function $\psi=\sum_{k=1}^{n} \mathbf{x}_{k} \chi_{E_{k}}$, we let

$$
\int_{E} \psi d m=\sum_{k=1}^{n} m\left(E_{k} \cap E\right) \mathbf{x}_{k} .
$$

A function $f: T \rightarrow \mathbb{X}$ is called measurable if there is a sequence $\left\langle f_{n}\right\rangle$ of simple integrable functions such that $\lim _{n \rightarrow \infty} f_{n}(t)=f(t)$ for each $t \in T$. A measurable function $f: T \rightarrow \mathbb{X}$ is called the Dobrakov integrable if there is a sequence $\left\langle f_{n}\right\rangle$ of simple integrable functions converging almost everywhere $\hat{m}$ to $f$. In this case, the integral of the function $f$ on a set $E$ in $\mathcal{K}$ is defined by the equality

$$
\text { (D) }-\int_{E} f d m=\lim _{n \rightarrow \infty} \int_{E} f_{n} d m
$$

Here this limit is uniform with respect to $E$ in $\mathcal{K}$.

(H) Let $(\Omega, \mathscr{B}, \mu)$ be a measure space. Let $X: \Omega \rightarrow \mathbb{R}^{n+1}$ be a measurable function and $F$ a $\mathbb{C}$-valued integrable function on $(\Omega, \mathcal{B}, \mu)$. Let $P_{X}(A)=\mu\left(X^{-1}(A)\right)$ for $A \in \mathcal{B}\left(\mathbb{R}^{n+1}\right)$. Then $P_{X}$ is a measure on $\mathcal{B}\left(\mathbb{R}^{n+1}\right)$. By the RadonNikodym theorem, there is a function $E^{\mu}(F \mid X)$, unique up to $\mu$-null sets such that 


$$
\int_{X^{-1}(A)} F d \mu=\int_{A} E^{\mu}(F \mid X) d P_{X}
$$

for $A \in \mathscr{B}\left(\mathbb{R}^{n+1}\right)$. This function $E^{\mu}(F \mid X)$ is called the conditional expectation of $F$ given $X$.

(I) Let $\varphi$ be in $\mathcal{M}(\mathbb{R})$ and $\eta$ be a complex-valued Borel measure on $[a, b]$. A complex-valued Borel measurable function $\theta$ on $[a, b] \times \mathbb{R}$ is said to belong to $L_{\varphi ; \infty, 1 ; \eta}\left(\right.$ or $\left.L_{\varphi ; \infty, 1 ; \eta}^{t}\right)$ if

$$
\|\theta\|_{\varphi ; \infty, 1 ; \eta}=\int_{[a, b]}\|\theta(s, \cdot)\|_{\varphi ; \infty} d|\eta|(s)
$$

is finite where $\|\theta(0, \cdot)\|_{\varphi ; \infty}$ is $\inf \{\lambda>0|| \varphi \mid(\{\xi$ in $\mathbb{R}|| \theta(0, \xi) \mid>\lambda\})=0\}$ and $\|\theta(s, \cdot)\|_{\varphi ; \infty}$ is $\inf \{\lambda>$ $0 \mid m_{L}(\{\xi$ in $\left.\mathbb{R}|| \theta(s, \xi) \mid>\lambda\})=0\right\}$ for $0<s \leq t$. If $\theta$ is bounded Borel measurable then $\theta$ is in $L_{\varphi ; \infty, 1 ; \eta}$.

(J) For $\theta$ be in $L^{\infty}\left(\mathbb{R}, m_{L}\right)$, we consider an operator $M_{\theta}$ from $\mathcal{R} \mathcal{M}(\mathbb{R})$ into itself such that

$$
\left[M_{\theta}(\mu)\right](E)=\int_{E} \frac{d \mu}{d m_{L}}(\xi) \theta(\xi) d m_{L}(\xi)
$$

for $E$ in $\mathscr{B}(\mathbb{R})$ and for $\mu$ in $\mathcal{R M}(\mathbb{R})$. Then

$$
\frac{d M_{\theta}(\mu)}{d m_{L}}(\xi)=\frac{d \mu}{d m_{L}}(\xi) \theta(\xi)
$$

so $M_{\theta}$ is well-defined. Since $\left|M_{\theta}(\mu)\right|(\mathbb{R}) \leq \int_{\mathbb{R}}\left|\frac{d \mu}{d m_{L}}(\xi)\right||\theta(\xi)| d m_{L}(\xi) \leq\|\theta\|_{\infty}|\mu|(\mathbb{R}), M_{\theta}$ is a bounded linear operator.

For $s>0$, we let

$$
P_{s}(E)=\int_{E} \frac{1}{\sqrt{2 \pi s}} \exp \left\{-\frac{u^{2}}{2 s}\right\} d m_{L}(u)
$$

for $E$ in $\mathcal{B}(\mathbb{R})$.

For $s>0$, we consider an operator $S_{s}$ from $\mathcal{R} \mathcal{M}(\mathbb{R})$ into itself such that

$$
\begin{aligned}
{\left[S_{s}(\mu)\right](E) } & =\left(\mu * P_{s}\right)(E) \\
& =\frac{1}{\sqrt{2 \pi s}} \int_{\mathbb{R}}\left[\int_{E} \exp \left\{-\frac{(u-v)^{2}}{2 s}\right\} d m_{L}(u)\right] d \mu(v) .
\end{aligned}
$$

Then

$$
\frac{d S_{S}(\mu)}{d m_{L}}(\xi)=\frac{1}{\sqrt{2 \pi s}} \int_{\mathbb{R}} \exp \left\{-\frac{(\xi-v)^{2}}{2 s}\right\} d \mu(v),
$$

so $S_{s}$ is well-defined. It is not hard to show that $S_{s}$ is a bounded linear operator and the operator norm $\left\|S_{s}\right\|$ of $S_{s}$ is less than or equals one.

Let $s_{1}$ and $s_{2}$ be two positive real numbers. Then by the Chapman-Kolmogorov equation in [17] and the classical Fubini theorem, we have

$$
S_{s_{1}} \circ S_{s_{2}}=S_{s_{1}+s_{2}} .
$$

For $s>0$, for $\varphi$ in $\mathcal{M}(\mathbb{R})$, for a Borel measurable $|\varphi|$-essentially bounded function $\theta$ on $(\mathbb{R}, \mathcal{B}(\mathbb{R}))$ and for $E$ in $\mathcal{B}(\mathbb{R})$, we let

$$
[T(s, \varphi, \theta)](E)=\frac{1}{\sqrt{2 \pi s}} \int_{\mathbb{R}}\left[\int_{E} \theta(v) \exp \left\{-\frac{(u-v)^{2}}{2 s}\right\} d m_{L}(u)\right] d \varphi(v) .
$$

Then $T(s, \varphi, \theta)$ is in $\mathcal{R} \mathcal{M}(\mathbb{R})$ and

$$
\frac{d T(s, \varphi, \theta)}{d m_{L}}(u)=\frac{1}{\sqrt{2 \pi s}} \int_{\mathbb{R}} \theta(v) \exp \left\{-\frac{(u-v)^{2}}{2 s}\right\} d \varphi(v) .
$$

(K) Let $\varphi$ is a measure on $(\mathbb{R}, \mathscr{B}(\mathbb{R}))$ and let $F: C[a, b] \rightarrow \mathbb{R}$ be a measurable function. For all $\lambda>0$, if the integral $\int_{C[a, b]} F\left(\lambda^{-1} x\right) d \omega_{\varphi}(x)$ exists, then we denote

$$
\int_{C[a, b]} F\left(\lambda^{-1} x\right) d \omega_{\varphi}(x)=J(\lambda)
$$

And if there exists a function $J^{*}(\lambda)$ analytic in the half-plane $\mathbb{C}^{+}$such that $J(\lambda)=J^{*}(\lambda)$ for almost all real $\lambda>0$, then we write

$$
\int_{C[a, b]}^{a n a n w_{\lambda}} F(x) d \omega_{\varphi}(x)=J^{*}(\lambda)
$$

and we call that $J^{*}(\lambda)$ is the analytic analogue of Wiener integral of $F$ over $C[a, b]$ with parameter $\lambda$. And for non-zero 
real number $q$, if the limit $\lim _{\substack{\lambda \rightarrow-i q \\ \lambda \in \mathbb{C}^{+}}} J^{*}(\lambda)$ exists, then we denote

$$
\lim _{\substack{\lambda \rightarrow-i q \\ \lambda \in \mathbb{C}^{+}}} J^{*}(\lambda)=\int_{C[a, b]}^{\text {an anf }} \underset{ }{ } F(x) d \omega_{\varphi}(x)
$$

and we say that the limit is the analytic analogue of Feynman integral of $F$.

Notation. For $\lambda \in \mathbb{C}^{+}$and $y \in C[a, b]$ let

$$
\left(T_{a n, \lambda} F\right)(y)=\int_{C[a, b]}^{a n a n w_{\lambda}} F(x+y) d \omega_{\varphi}(x) .
$$

And given a number $p$ such that $1 \leq p \leq \infty, p$ and $p^{\prime}$ will always be related by $\frac{1}{p}+\frac{1}{p^{\prime}}=1$. Let $\left\{H_{n}\right\}$ and $H$ be analogue of Wiener measurable functions such that for each $\rho>0$,

$$
\left.\lim _{n \rightarrow \infty} \int_{C[a, b]} \mid H_{n}(\rho y)-H(\rho y)\right)\left.\right|^{2} d \omega_{\varphi}(y)=0 .
$$

Then we write

$$
\lim _{n \rightarrow \infty}\left(w_{\varphi, s}^{2}\right) H_{n} \stackrel{a n}{\approx} H
$$

and we call $H$ the scale invariant limit in the mean of order 2 of $H_{n}$ over $C[a, b]$. We define a similar definition for any real number instead of $n$. Let $q$ be non-zero real number. For $1<p \leq 2$ we define the $L_{p}$ analytic Fourier-Feynman transform of $F$, which we denote by $T_{a n, q}^{(p)} F$, by the formula

$$
\left(T_{a n, q}^{(p)} F\right)(y)=\lim _{\substack{\lambda \rightarrow-i q \\ \lambda \in \mathbb{C}^{+}}}\left(w_{\varphi, s}^{p^{\prime}}\right)\left(T_{a n, \lambda} F\right)(y)
$$

whenever this limit exists. Let $F$ be a functional on analogue of Wiener space such that $\left(T_{a n, \lambda} F\right)(y)$ exists in $\mathbb{C}^{+}$for $s$-almost every $y$. We define the $L_{1}$ analytic analogue of Fourier-Feynman transform of $F$, which we denote by $T_{a n, q}^{(1)} F$, as that functional (if it exists) on analogue of Wiener space such that

$$
\left(T_{a n, q}^{(1)} F\right)(y)=\lim _{\substack{\lambda \rightarrow-i q \\ \lambda \in \mathbb{C}^{+}}}\left(T_{a n, \lambda} F\right)(y)
$$

for $s$-almost every $y$. For each natural number $n$ and a partition $a=t_{0}<t_{1}<\cdots<t_{n}=b$, let $\mathcal{A}_{n}$ be the collection of functions $F: C[0, t] \rightarrow \mathbb{R}$ satisfying (1) and (2) below;

(1) $f$ is a measurable function on $\mathbb{R}^{n+1}$.

(2) $\left.F(x) \stackrel{a n}{\approx} f\left(x_{0}\right), x\left(t_{1}\right), \ldots, x\left(t_{n}\right)\right)$.

\section{The complex-valued analogue of Wiener measure $\omega_{\varphi}$}

In this section, we will introduce a complex-valued analogue of Wiener measure $\omega_{\varphi}$ on $C[a, b]$ and we will give some examples of it.

Let $n$ be a non-negative integer. For $\vec{t}=\left(t_{0}, t_{1}, \ldots, t_{n}\right)$ with $a=t_{0}<t_{1}<\cdots<t_{n} \leq b$, let $J_{\vec{t}}: C[a, b] \rightarrow \mathbb{R}^{n+1}$ be a function with

$$
J_{\vec{t}}(x)=\left(x\left(t_{0}\right), x\left(t_{1}\right), \ldots, x\left(t_{n}\right)\right) .
$$

For $B_{j}(j=0,1,2, \ldots, n)$ in $\mathcal{B}(\mathbb{R})$, the subset $J_{\vec{t}}^{-1}\left(\prod_{j=0}^{n} B_{j}\right)$ of $C[a, b]$ is called an interval and let $\ell$ be the set of all intervals. For a non-negative finite Borel measure $\varphi$ on $(\mathbb{R}, \mathscr{B}(\mathbb{R}))$, we let

$$
\begin{aligned}
m_{\varphi}\left(J_{\vec{t}}^{-1}\left(\prod_{j=0}^{n} B_{j}\right)\right) \\
\quad=\int_{B_{0}}\left[\int_{\prod_{j=1}^{n} B_{j}} W\left(n+1 ; \vec{t} ; u_{0}, u_{1}, \ldots, u_{n}\right) d \prod_{j=1}^{n} m_{L}\left(u_{1}, \ldots, u_{n}\right)\right] d \varphi\left(u_{0}\right)
\end{aligned}
$$

where

$$
W\left(n+1 ; \vec{t} ; u_{0}, u_{1}, \ldots, u_{n}\right)=\left(\prod_{j=1}^{n} \frac{1}{\sqrt{2 \pi\left(t_{j}-t_{j-1}\right)}}\right) \exp \left\{-\frac{1}{2} \sum_{j=1}^{n} \frac{\left(u_{j}-u_{j-1}\right)^{2}}{t_{j}-t_{j-1}}\right\}
$$

Then $\mathcal{B}(C[a, b])$, the set of all Borel subsets in $C[a, b]$, coincides with the smallest $\sigma$-algebra generated by $\ell$ and there exists a unique positive measure $\omega_{\varphi}$ on $(C[a, b], \mathcal{B}(C[a, b]))$ such that $\omega_{\varphi}(I)=m_{\varphi}(I)$ for all $I$ in $\ell$. 
For $\varphi$ in $\mathcal{M}(\mathbb{R})$ with the Jordan decomposition $\varphi=\sum_{j=1}^{4} \alpha_{j} \varphi_{j}$, let $\omega_{\varphi}=\sum_{j=1}^{4} \alpha_{j} \omega_{\varphi_{j}}$. We say that $\omega_{\varphi}$ is the complexvalued analogue of Wiener measure on $(C[a, b], \mathcal{B}(C[a, b]))$, associated with $\varphi$. If $\varphi$ is a Dirac measure $\delta_{0}$ at the origin in $\mathbb{R}$ then $\omega_{\varphi}$ is the classical Wiener measure.

By the change of variables formula, we can easily prove the following theorem.

Theorem 3.1 (The Wiener integration formula). If $f: \mathbb{R}^{n+1} \rightarrow \mathbb{C}$ is a Borel measurable function then the following equality holds.

$$
\begin{aligned}
& \int_{C[a, b]} f\left(x\left(t_{0}\right), x\left(t_{1}\right), \ldots, x\left(t_{n}\right)\right) d \omega_{\varphi}(x) \\
& \stackrel{*}{=} \int_{\mathbb{R}^{n+1}} f\left(u_{0}, u_{1}, \ldots, u_{n}\right) W\left(n+1 ; \vec{t} ; u_{0}, u_{1}, \ldots, u_{n}\right) \\
& \quad d\left(\prod_{j=1}^{n} m_{L} \times \varphi\right)\left(\left(u_{1}, u_{2}, \ldots, u_{n}\right), u_{0}\right)
\end{aligned}
$$

where $\stackrel{*}{=}$ means that if one side exists then both sides exist and the two values are equal.

Remark 3.2. Let $\varphi$ be in $\mathcal{M}(\mathbb{R})$.

(1) It is not hard to show that $\omega_{\varphi}$ has no atoms.

(2) $\omega_{\varphi}(C[a, b])=\varphi(\mathbb{R})$.

(3) Let $J_{t}: C[a, b] \rightarrow \mathbb{C}$ be a function with $J_{t}(x)=x(t)$. Then for $E$ in $\mathcal{B}(\mathbb{R}), \omega_{\varphi}\left(J_{t}^{-1}(E)\right)=\left[S_{t}(\varphi)\right](E)$.

Example 3.3. Let $\varphi$ be in $\mathcal{M}(\mathbb{R})$.

(1) Let $I=\{x$ in $C[0, t] \mid x(0)$ is in $B\}$ where $B$ is in $\mathbb{B}(\mathbb{R})$. Then $\omega_{\varphi}(I)=\varphi(B)$.

(2) Suppose that $f(u)=u$ is $\varphi$-integrable. Then for $0 \leq s \leq t$,

$$
\int_{C[0, t]} x(s) d \omega_{\varphi}(x)=\int_{\mathbb{R}} u d \varphi(u) .
$$

If $\varphi=\delta_{p}$ then $\int_{C[0, t]} x(s) d \omega_{\varphi}(x)=p$ and if $\varphi$ has a normal distribution with mean $\alpha$ and variation $\sigma^{2}$ then $\int_{C[0, t]} x(s) d \omega_{\varphi}(x)=\alpha$.

(3) Suppose that $g(u)=u^{2}$ is $\varphi$-integrable. Then for $0 \leq s \leq t$,

$$
\int_{C[0, t]} x(s)^{2} d \omega_{\varphi}(x)=\int_{\mathbb{R}} u^{2} d \varphi(u)+s \varphi(\mathbb{R}) .
$$

If $\varphi=\delta_{p}$ then $\int_{C[0, t]} x(s)^{2} d \omega_{\varphi}(x)=p^{2}+s$ and if $\varphi$ has a normal distribution with mean $\alpha$ and variance $\sigma^{2}$ then

$$
\int_{C[0, t]} x(s)^{2} d \omega_{\varphi}(x)=\alpha^{2}+\sigma^{2}+s .
$$

(4) Let $\mathcal{F}(\varphi)$ be the Fourier transform of a measure $\varphi$, that is, $[\mathcal{F}(\varphi)](\xi)=\int_{\mathbb{R}} \exp \{i \xi u\} d \varphi(u)$. Then for $0 \leq s \leq t$,

$$
\int_{C[0, t]} \exp \{i \xi x(s)\} d \omega_{\varphi}(x)=\exp \left\{-\frac{s \xi^{2}}{2}\right\}[\mathcal{F}(\varphi)](\xi) .
$$

If $\varphi=\delta_{p}$ then $\int_{C[0, t]} \exp \{i \xi x(s)\} d \omega_{\varphi}(x)=\exp \left\{-\frac{s \xi^{2}}{2}+i p \xi\right\}$ and if $\varphi$ has a normal distribution with mean $\alpha$ and variance $\sigma^{2}$ then

$$
\int_{C[0, t]} \exp \{i \xi x(s)\} d \omega_{\varphi}(x)=\exp \left\{-\frac{\left(s+\sigma^{2}\right) \xi^{2}}{2}+i \alpha \xi\right\} .
$$

Let $0<s \leq t$ be given and let $J_{s}: C[0, t] \rightarrow \mathbb{R}$ be a function with $J_{s}(x)=x(s)$. We assume that $\left\langle\varphi_{n}\right\rangle$ converges to $\varphi$ weakly. By calculation similar as in this example, since $\left\langle\mathcal{F}\left(\varphi_{n}\right)\right\rangle$ converges to $\mathcal{F}(\varphi)$ pointwise, $\left\langle\mathcal{F}\left(\omega_{\varphi_{n}}\left(J_{s}^{-1}(\cdot)\right)\right)\right\rangle$ converges to $\mathcal{F}\left(\omega_{\varphi}\left(J_{s}^{-1}(\cdot)\right)\right)$ pointwise, so by the continuity theorem in [1, Theorem 12-5A, p 273], $\left\langle\omega_{\varphi_{n}}\left(J_{s}^{-1}(\cdot)\right)\right\rangle$ converges to $\omega_{\varphi}\left(J_{s}^{-1}(\cdot)\right)$ weakly.

(5) We assume that $k(u)=u^{2}$ is $\varphi$-integrable. For $0 \leq s_{1}, s_{2} \leq t$,

$$
\int_{C[0, t]} x\left(s_{1}\right) x\left(s_{2}\right) d \omega_{\varphi}(x)=\left(\min \left\{s_{1}, s_{2}\right\}\right) \varphi(\mathbb{R})+\int_{\mathbb{R}} u^{2} d \varphi(u) .
$$

If $\varphi=\delta_{p}$ then $\int_{C[0, t]} x\left(s_{1}\right) x\left(s_{2}\right) d \omega_{\varphi}(x)=\min \left\{s_{1}, s_{2}\right\}+p^{2}$ and if $\varphi$ has a normal distribution with mean $\alpha$ and variance $\sigma^{2}$.

$$
\int_{C[0, t]} x\left(s_{1}\right) x\left(s_{2}\right) d \omega_{\varphi}(x)=\min \left\{s_{1}, s_{2}\right\}+\alpha^{2}+\sigma^{2} .
$$

(6) For $0 \leq s_{1}<s_{2} \leq s_{3}<s_{4} \leq t$ and for $\alpha, \beta$ in $\mathbb{R}$, using the change of variable formula, we have 


$$
\begin{gathered}
\varphi(\mathbb{R}) \omega_{\varphi}\left(\left\{x \text { in } C[0, t] \mid x\left(s_{2}\right)-x\left(s_{1}\right) \leq \alpha \text { and } x\left(s_{4}\right)-x\left(s_{3}\right) \leq \beta\right\}\right) \\
=\omega_{\varphi}\left(\left\{x \text { in } C[0, t] \mid x\left(s_{2}\right)-x\left(s_{1}\right) \leq \alpha\right\}\right) \\
\cdot \omega_{\varphi}\left(\left\{x \text { in } C[0, t] \mid x\left(s_{4}\right)-x\left(s_{3}\right) \leq \beta\right\}\right) .
\end{gathered}
$$

Hence, if $\varphi$ is a probability measure then $x\left(s_{2}\right)-x\left(s_{1}\right)$ and $x\left(s_{4}\right)-x\left(s_{3}\right)$ are independent.

Theorem 3.4. For $\varphi$ in $\mathcal{M}(\mathbb{R})$, $\left|\omega_{\varphi}\right|=\omega_{|\varphi|}$ on $(C[a, b], \mathcal{B}(C[a, b]))$.

We consider a set $\mathcal{A}=\left\{E\right.$ in $\left.\mathcal{B}(C[a, b])|| \omega_{\varphi} \mid(E)=\omega_{|\varphi|}(E)\right\}$. Then we have $\ell \subset \mathcal{A}$. Since $\left|\omega_{\varphi}\right|$ and $\omega_{|\varphi|}$ are both measures on $(C[a, b], \mathcal{B}(C[a, b])),\left|\omega_{\varphi}\right|=\omega_{|\varphi|}$ on $\mathcal{B}(C[a, b])$.

Theorem 3.5. If a sequence $\left\langle\varphi_{n}\right\rangle$ of non-negative finite measures, converges to $\varphi$ in the sense of total variation norm then a sequence $\left\langle\omega_{\varphi_{n}}\right\rangle$ converges to $\omega_{\varphi}$ in the total variation norm.

From [2], we can find a sequence $\left\langle P_{n}\right\rangle$ of measures on $C[a, b]$ such that $\left\langle P_{n}\right\rangle$ doesnt converges to $P$ weakly even though every finite dimensional measures of $P_{n}$ converges to some finite dimensional measure of $P$ weakly. Here, we want to find the conditions such that $\left\langle\omega_{\varphi_{n}}\right\rangle$ converges to $\omega_{\varphi}$ weakly whenever $\left\langle\varphi_{n}\right\rangle$ converges to $\varphi$ weakly.

Lemma 3.6. Let $X:[a, b] \times C[a, b] \rightarrow \mathbb{R}$ be a function with $X(s, x)=x(s)$. Then for $a<t_{1} \leq b$ and for $\epsilon>0$,

$$
\omega_{\varphi}\left(\left\{x \mid \sup \left\{x(s)-x(a) \mid a \leq s \leq t_{1}\right\} \geq \epsilon\right\}\right) \leq \frac{1}{\epsilon} \sqrt{\frac{2 t_{1}}{\pi}} \exp \left\{-\frac{\epsilon^{2}}{2 t_{1}}\right\} .
$$

Lemma 3.7. For $\epsilon>0$ and $\lambda>0$,

$$
\omega_{\varphi}\left(\left\{x\left|\sup _{0 \leq t \leq \epsilon}\right| x(t)-x(\epsilon) \mid \leq \lambda\right\}\right)=\omega_{\varphi}\left(\left\{x\left|\sup _{0<t<\frac{\epsilon}{2}}\right| x(t)-x(0) \mid \leq \lambda\right\}\right)^{2}
$$

\section{Corollary 3.8.}

$$
\omega_{\varphi}\left(\left\{x\left|\sup _{0 \leq s \leq t_{1}}\right| x(s)-x\left(t_{1}\right) \mid>\lambda\right\}\right) \leq \frac{1}{\lambda} \sqrt{\frac{t_{1}}{\pi}} e^{-\frac{\lambda^{2}}{t_{1}}}\left(2-\frac{1}{\lambda} \sqrt{\frac{t_{1}}{\pi}} e^{-\frac{\lambda^{2}}{t_{1}}}\right) .
$$

Corollary 3.9. For each positive $\epsilon$ and $\eta$, there exists a $\delta$ with $0<\delta<1$ such that for $s_{1}, s_{2}$ in $[a, b]$

$$
\omega_{\varphi}\left(\left\{x\left|\sup _{\left|s_{1}-s_{2}\right|<\delta}\right| x\left(s_{1}\right)-x\left(s_{2}\right) \mid \geq \epsilon\right\}\right) \leq \eta .
$$

From [2], we find the following theorem.

Theorem 3.10. The sequence $\left\langle P_{n}\right\rangle$ of probability measures on $C[a, b]$ is tight, that is, for positive $\epsilon$ there exists a compact set $K$ such that $P_{n}(K)>1-\epsilon$ for all natural number $n$, if and only if

(i) for each positive $\eta$, there exists an $\alpha$ such that $P_{n}(\{x|| x(a) \mid>\alpha\}) \leq \eta$ for all $n$ and

(ii) for each positive $\epsilon$ and $\eta$, there exists a $\delta$ with $0<\delta<1$ and a natural number $n_{0}$ such that for $n \geq n_{0}$,

$$
P_{n}\left(\left\{x\left|\sup _{\left|s_{1}-s_{2}\right|<\delta}\right| x\left(s_{1}\right)-x\left(s_{2}\right) \mid \geq \epsilon\right\}\right) \leq \eta .
$$

From [2], we can find a sequence $\left\langle P_{n}\right\rangle$ of measures on $C[a, b]$ such that $\left\langle P_{n}\right\rangle$ doesnt converges to $P$ weakly even though every finite dimensional measures of $P_{n}$ converges to some finite dimensional measure of $P$ weakly. Here, we want to find the conditions such that $\left\langle\omega_{\varphi_{n}}\right\rangle$ converges to $\omega_{\varphi}$ weakly whenever $\left\langle\varphi_{n}\right\rangle$ converges to $\varphi$ weakly.

Theorem 3.11. Let $P_{n}, P$ be probability measures on $(C[a, b], \mathcal{B}(C[a, b]))$. If the finite dimensional distributions of $P_{n}$ converge weakly to those of $P$, and if $\left\langle P_{n}\right\rangle$ is tight, then $\left\langle P_{n}\right\rangle$ converges to $P$ weakly.

Theorem 3.12. Suppose $\left\langle\varphi_{n}\right\rangle$ is tight. Then $\left\langle\omega_{\varphi_{n}}\right\rangle$ is also tight.

Lemma 3.13. Let $f: \mathbb{R}^{n+1} \rightarrow \mathbb{R}$ be bounded continuous. Let $\vec{t}=\left(t_{0}, t_{1}, \ldots, t_{n}\right)$ be a vector in $\mathbb{R}^{n+1}$ with $t_{0}=a<t_{1}<\cdots<t_{n} \leq b$ and let $J_{\vec{t}}: C[a, b] \rightarrow \mathbb{R}^{n}$ be a function with $J_{\vec{t}}(x)=\left(x\left(t_{0}\right), x\left(t_{1}\right), \ldots, x\left(t_{n}\right)\right)$. Suppose $\left\langle\varphi_{n}\right\rangle$ converges to $\varphi$ weakly. Then

$$
\begin{gathered}
\lim _{m \rightarrow \infty} \int_{\mathbb{R}^{n+1}} f\left(u_{0}, u_{1}, \ldots, u_{n}\right) d \omega_{\varphi_{m}} J_{\vec{t}}^{-1}(x) \\
=\lim _{m \rightarrow \infty} \int_{C[a, b]} f\left(J_{\vec{t}}(x)\right) d \omega_{\varphi_{m}}(x)
\end{gathered}
$$




$$
\begin{aligned}
= & \lim _{m \rightarrow \infty} \int_{\mathbb{R}} \int_{\mathbb{R}^{n}} f\left(u_{0}, u_{1}, \ldots, u_{n}\right) \frac{1}{\prod_{j=1}^{n} \sqrt{2 \pi\left(t_{j}-t_{j-1}\right)}} \\
& \exp \left\{-\sum_{j=1}^{n} \frac{\left(u_{j}-u_{j-1}\right)^{2}}{2\left(t_{j}-t_{j-1}\right)}\right\} d \prod_{j=1}^{n} m_{L}\left(u_{1}, \ldots, u_{n}\right) d \varphi_{m}\left(u_{0}\right) \\
= & \int_{C[a, b]} f\left(J_{\hat{t}}(x)\right) d \omega_{\varphi}(x) .
\end{aligned}
$$

Theorem 3.14. If $\left\langle\varphi_{n}\right\rangle$ is tight and $\left\langle\varphi_{n}\right\rangle$ converges to $\varphi$ weakly, $\left\langle\omega_{\varphi_{n}}\right\rangle$ converges to $\omega_{\varphi}$ weakly.

Remark 3.15. The referee pointes out the following facts: for $y$ in $C[a, b]$, there are $\alpha$ in $\mathbb{R}$ and $x$ in $C_{0}[a, b]$ with $y=\alpha+x$ where $\alpha=y(a)$ and $x=y-\alpha$ is in $C_{0}[a, b]$. Let $\psi: C[a, b] \rightarrow \mathbb{R} \oplus C_{0}[a, b]$ be a function with $\psi(y)=(\alpha, x)$ as in above. Then $\|y\|_{\infty} \leq|\alpha|+\|x\|_{\infty} \equiv\|(\alpha, x)\|=\|\psi(y)\|$. By Two norm theorem [23], $\psi$ is a homeomophism. So we have $\omega_{\varphi}=\left(\varphi \times m_{\omega}\right) \circ \psi^{-1}$. Using this facts, we can easily prove the following corollary.

Corollary 3.16. Let $f$ be in $L_{1}(\mathbb{R})$ and let $\varphi(E)=\int_{E} f(x) d m_{L}(x)$ where $f>0$ and $E$ is a Borel subset of $\mathbb{R}$. For any integrable function $F$,

$$
\begin{aligned}
& \int_{C[a, b] \times C[a, b]} F(x, y) d \omega_{\varphi} \times \omega_{\varphi}(x, y) \\
& \quad=\int_{C[a, b] \times C[a, b]} F(x \cos \theta-y \sin \theta, x \sin \theta+y \cos \theta) d \omega_{\varphi} \times \omega_{\varphi}(x, y),
\end{aligned}
$$

for all real number $\theta$ if and only if the function $f(x)$ has the form $A e^{-a x^{2}}$ where $A$ and a are positive constants.

\section{A translation theorem on $\left(C[a, b], \mathscr{B}(C[a, b]), \omega_{\varphi}\right)$ and the Paley-Wiener-Zygmund integral}

It is well-known fact that there is no quasi-invariant probability measure on the infinite dimensional vector space [39]. So, there is no quasi-invariant probability measure on $C_{0}[a, b]$ or $C[a, b]$. In 1944, under the some assumptions, Cameron and Martin established a translation theorem on $\left(C_{0}[a, b], m_{w}\right)$ in [4]. In this section, we will prove a translation theorem on $\left(C[a, b], \omega_{\varphi}\right)$ under the similar assumptions to Cameron's assumptions. From these concepts, we will show that the Paley-Wiener-Zygmund integral is well-defined $\omega_{\varphi}$-a.e. By either the similar method as in the proof of Cameron and Martin's translation theorem on $C_{0}[a, b]$ in [4] or Remark 3.15, we can prove the following theorem.

Theorem 4.1 (The translation theorem on $\left.\left(C[a, b], \mathcal{B}(C[a, b]), \omega_{\varphi}\right)\right)$. Let $h$ be in $C[a, b]$ and of bounded variation. Let $\alpha$ be in $\mathbb{R}$ and let

$$
x_{0}(s)=\int_{a}^{s} h(u) d m_{L}(u)+\alpha
$$

for $a \leq s \leq b$. Let $L: C[a, b] \rightarrow C[a, b]$ be a function with $L(x)=x+x_{0}$ and let $\varphi$ be a probability measure on $(\mathbb{R}, \mathcal{B}(\mathbb{R}))$. Let $\varphi_{\alpha}$ be a measure on $(\mathbb{R}, \mathcal{B}(\mathbb{R}))$ such that $\varphi_{\alpha}(B)=\varphi(B+\alpha)$ for $B$ in $\mathcal{B}(\mathbb{R})$ and $\varphi_{\alpha} \ll \varphi$. Then if $F$ is $\omega_{\varphi^{-}}$ integrable then $F\left(x+x_{0}\right)$ is $\omega_{\varphi}$-integrable of $x$ and

$$
\begin{aligned}
& \int_{C[a, b]} F(y) d \omega_{\varphi}(y) \\
& \quad=e^{-\frac{1}{2}\|h\|_{2}^{2}} \int_{C[a, b]} F\left(x+x_{0}\right) e^{-\int_{a}^{b} h(u) d x(u)} \frac{d \varphi_{\alpha}}{d \varphi}(x(0)) d \omega_{\varphi}(x) .
\end{aligned}
$$

Putting $F \equiv 1$ in Theorem 4.1, we have the following corollary.

Corollary 4.2. Under the assumptions in Theorem 4.1,

$$
\int_{C[a, b]} \exp \left\{-\int_{a}^{b} h(u) d x(u)\right\} d \omega_{\varphi}(x)=\exp \left\{-\frac{1}{2}\|h\|_{2}^{2}\right\} .
$$

Replacing $h$ by $\lambda h$ in the above Corollary 4.2, by the uniqueness theorem for analytic extension in the theory of complex analysis, we have the following corollary.

Corollary 4.3. Under the assumptions in Theorem 4.1, for all $\lambda$ in $\mathbb{C}$, 


$$
\int_{C[a, b]} \exp \left\{-\lambda \int_{a}^{b} h(u) d x(u)\right\} d \omega_{\varphi}(x)=\exp \left\{-\frac{\lambda^{2}}{2}\|h\|_{2}^{2}\right\} .
$$

Theorem 4.4. Under the assumptions in Theorem 4.1, consider a random variable $X: C[a, b] \rightarrow \mathbb{R}$ with $X(x)=\int_{a}^{b} h(u) d x(u)$. Then $X$ has a normal distribution with the mean zero and the variation $\|h\|_{2}^{2}$.

By the same method as in the proof of Theorem 29.7 in [41], we can prove the following theorem.

Theorem 4.5. Let $\left\{h_{1}, h_{2}, \ldots, h_{n}\right\}$ be an orthonormal system such that each $h_{i}$ is of bounded variation. For $i=1,2, \ldots, n$, let $X_{i}(x)=\int_{a}^{b} h_{i}(s) d x(s)$. Then $X_{1}, X_{2}, \ldots, X_{n}$ are independent, each $X_{i}$ has the standard normal distribution. Moreover, if $f: \mathbb{R}^{n} \rightarrow \mathbb{R}$ is Borel measurable,

$$
\begin{aligned}
& \int_{C[a, b]} f\left(X_{1}(x), X_{2}(x), \ldots, X_{n}(x)\right) d \omega_{\varphi}(x) \\
& \quad \stackrel{*}{=}(2 \pi)^{-\frac{n}{2}} \int_{\mathbb{R}^{n}} f\left(u_{1}, u_{2}, \ldots, u_{n}\right) \exp \left\{-\frac{1}{2} \sum_{j=1}^{n} u_{j}\right\} d \prod_{i=1}^{n} m_{L}\left(u_{1}, u_{2}, \ldots, u_{n}\right)
\end{aligned}
$$

where $\stackrel{*}{=}$ means that if one side exists then both sides exist and the two values are equal.

Let $\left\{h_{k} \mid k=1,2, \ldots\right\}$ be a complete orthonormal set in $L^{2}\left([a, b], m_{L}\right)$ such that each $h_{k}$ is of bounded variation. For $f$ in $L^{2}\left([a, b], m_{L}\right)$ and $x$ in $C[a, b]$, we let

$$
\int_{a}^{b} f(s) \hat{d x}(s)=\lim _{n \rightarrow \infty} \int_{a}^{b}\left[\sum_{k=1}^{n} \int_{a}^{b} f(u) e_{k}(u) d m_{L}(u) e_{k}(v)\right] d x(v)
$$

if the limit exists. Then

$$
\int_{a}^{b} f(s) \hat{d x}(s)
$$

is called the Paley-Wiener-Zygmund integral of $f$ according to $x$. By the routine method in the theory of Wiener space, we can prove that the integral $\int_{a}^{b} f(s) \hat{d x}(s)$ is independent on the orthonormal set $\left\{e_{k} \mid k=1,2, \ldots\right\}$ and the PaleyWiener-Zygmund integral exists $\omega_{\varphi}$-a.e. $x$ in $C[a, b]$.

Remark 4.6. In 1980, Cameron and Storvick introduced the definitions and some related theories of the spaces $S, S^{\prime}$ and $S^{\prime \prime}$ of Wiener functionals. If we replace $\left(C_{0}[a, b], m_{w}\right)$ by $\left(C[a, b], \omega_{\varphi}\right)$ in their paper, we can prove various results on $\left(C[a, b], \omega_{\varphi}\right)$ which are similar to Cameron and Storvick's results in [6].

\section{The relationship between conditional expectation and Bartle integral with respect to a vector measure $V_{\varphi}$}

In this section, we will show that the Bartle integral with respect to $V_{\varphi}$ can be written as the iterated integrals with respect to complex-valued measure. From this, we recognize the relation between the Bartle integral and the conditional expectation on $\left(C[a, b], \omega_{\varphi}\right)[31]$.

Let $\varphi$ be a probability measure on $(\mathbb{R}, \mathscr{B}(\mathbb{R}))$. Let $n$ be a non-negative integer. Let $X$ be a $\mathbb{R}^{n+1}$-valued measurable function on $\left(C[a, b], \mathcal{B}(C[a, b]), \omega_{\varphi}\right)$. We write $P_{X}$ for a measure on $\left(\mathbb{R}^{n+1}, \mathcal{B}\left(\mathbb{R}^{n+1}\right)\right)$ determined by $X$, that is, $P_{X}(E)=$ $\omega_{\varphi}\left(X^{-1}(E)\right)$ for $E$ in $\mathcal{B}\left(\mathbb{R}^{n+1}\right)$.

For $\varphi$ in $\mathcal{M}(\mathbb{R})$ and for $B$ in $\mathscr{B}(C[a, b])$, we let $\left[V_{\varphi}(B)\right](E)=\omega_{\varphi}\left(B \cap X^{-1}(E)\right)$. Then $V_{\varphi}$ is a measure-valued measure on $(C[a, b], \mathcal{B}(C[a, b]))$ in the total variation norm sense.

Theorem 5.1. Let $\varphi$ be a probability measure on $(\mathbb{R}, \mathcal{B}(\mathbb{R}))$ and let $f$ be bounded measurable on $(C[a, b], \mathscr{B}(C[a, b]))$. Then

$$
\left[(B a)-\int_{C[a, b]} f(x) d V_{\varphi}(x)\right](E)=\int_{E} E(f \mid X)(\xi) d P_{X}(\xi)
$$

for $\operatorname{E}$ in $\mathcal{B}\left(\mathbb{R}^{n+1}\right)$.

For a non-negative finite real valued measure in $\mathcal{M}(\mathbb{R})$, let $\varphi^{N}$ be a normalized measure of $\varphi$, that is, $\varphi^{N}(E)=\frac{\varphi(E)}{|\varphi|(\mathbb{R})}$ for $E$ in $\mathcal{B}(\mathbb{R})$ if $\varphi$ is a non-zero measure and $\varphi^{N}$ is a zero measure if $\varphi$ is a zero measure. For $\varphi$ in $\mathcal{M}(\mathbb{R})$ with the Jordan decomposition $\varphi=\sum_{j=1}^{4} \alpha_{j} \varphi_{j}, \omega_{\varphi}=\sum_{j=1}^{4} \alpha_{j} \omega_{\varphi_{j}}$ and for $j=1,2,3,4, \omega_{\varphi_{j}}=\left|\varphi_{j}\right|(\mathbb{R}) \varphi_{j}^{N}$. Hence, for $\varphi$ in $\mathcal{M}(\mathbb{R})$ with the Jordan decomposition $\varphi=\sum_{j=1}^{4} \alpha_{j} \varphi_{j}$, for $B$ in $\mathcal{B}(C[a, b])$ and for $E$ in $\mathscr{B}(\mathbb{R})$, 


$$
\begin{aligned}
{\left[V_{\varphi}(B)\right](E) } & =\omega_{\varphi}\left(B \cap J_{\vec{t}}^{-1}(E)\right) \\
& =\sum_{j=1}^{4} \alpha_{j} \omega_{\varphi_{j}}\left(B \cap J_{\vec{t}}^{-1}(E)\right) \\
& =\sum_{j=1}^{4} \alpha_{j}\left|\varphi_{j}\right|(\mathbb{R}) \omega_{\varphi_{j}^{N}}\left(B \cap J_{\vec{t}}^{-1}(E)\right) \\
& =\left[\sum_{j=1}^{4} \alpha_{j}\left|\varphi_{j}\right|(\mathbb{R}) V_{\varphi_{j}^{N}}(B)\right](E),
\end{aligned}
$$

so we have

$$
V_{\varphi}=\sum_{j=1}^{4} \alpha_{j}\left|\varphi_{j}\right|(\mathbb{R}) V_{\varphi_{j}^{N}} .
$$

Theorem 5.2. Let $\varphi$ in $\mathcal{M}(\mathbb{R})$, for a bounded measurable function $f$ on $(C[a, b], \mathcal{B}(C[a, b]))$ and $X(x)=x(b)$. Then

$$
\begin{aligned}
& {\left[(B a)-\int_{C[a, b]} f(x) d V_{\varphi}(x)\right](E)} \\
& \quad=\int_{E} E(f \mid X)(\xi) d P_{X}(\xi) \\
& \quad=\int_{E} E(f \mid X)(\xi) \frac{d P_{X}}{d m_{L}}(\xi) d m_{L}(\xi) \\
& \quad=\frac{1}{2 \pi} \int_{E} \int_{\mathbb{R}} e^{-i \xi u} \int_{C[a, b]} e^{i u x(t)} f(x) d \omega_{\varphi}(x) d m_{L}(u) d m_{L}(\xi)
\end{aligned}
$$

for $\operatorname{E}$ in $\mathcal{B}(\mathbb{R})$.

Remark 5.3. Putting $\varphi=\delta_{0}, \omega_{\varphi}=\omega$ and $X(x)=x(b)$, the classical Wiener measure and

$$
\begin{aligned}
& {\left[(B a)-\int_{C[a, b]} f(x) d V_{\varphi}(x)\right](E)} \\
& \quad=\frac{1}{2 \pi} \int_{E} \int_{\mathbb{R}} e^{-i \xi u} \int_{C[a, b]} f(x) d \omega_{\varphi}(x) d m_{L}(u) d m_{L}(\xi) \\
& \quad=\int_{E} E(f \mid X)(\xi) d m_{L}(\xi) \\
& \quad=\int_{X^{-1}(E)} f(x) d \omega(x) .
\end{aligned}
$$

Here $f$ is a bounded measurable function and $E$ is in $\mathcal{B}(\mathbb{R})$.

Theorem 5.4 (The Wiener integration formula for $V_{\varphi}$ ). Suppose for $k=1,2, \ldots, n, i_{k}$ is a nonnegative integer such that $m=n+\sum_{j=1}^{n} i_{j}+1$ and $a \equiv t_{0} \equiv t_{0,0}<t_{0,1}<t_{0,2}<\cdots<t_{0, i_{1}}<t_{1} \equiv t_{0, i_{1}+1} \equiv t_{1,0}<t_{1,1}<t_{1,2}<\cdots<$ $t_{n-1, i_{n}}<t_{n} \equiv t_{n-1, i_{n}+1} \equiv b$ and for $j=1,2, \ldots, n$. Let $X(x)=\left(x\left(t_{0}\right), x\left(t_{1}\right), \ldots, x\left(t_{n}\right)\right)$. If $f: \mathbb{B}^{m} \rightarrow \mathbb{R}$ is a Borel measurable function then the following equality holds;

$$
\begin{aligned}
& {\left[(B a)-\int_{C[a, b]} f\left(y\left(t_{0,0}\right), y\left(t_{0,1}\right), \ldots, y\left(t_{n-1, i_{n}+1}\right)\right) d V_{\varphi}^{J_{\vec{t}}}(y)\right](E)} \\
& \stackrel{*}{=} \int_{\mathbb{R}}\left[\int_{\mathbb{R}^{m-1}} f\left(u_{0,0}, u_{0,1}, \ldots, u_{n-1, i_{n}+1}\right) W_{m+1} \prod_{g=0}^{n} \chi_{E^{[g]}}\left(u_{g, 0}\right)\right. \\
& \left.\quad d\left(\prod_{i=1}^{m-1} \omega\right)\left(u_{0,0}, u_{0,1}, \ldots, u_{n-1, i_{n}+1}\right)\right] d m_{L}\left(u_{0,0}\right),
\end{aligned}
$$

where $E^{[g]}$ is the $g^{\text {th }}$-section of $E$.

\section{The simple formula for conditional expectation on analogue of Wiener measure space}

In this section, we prove the simple formula for conditional expectation on analogue of Wiener measure. Throughout in this section, let $a=t_{0}<t_{1}<\cdots<t_{n}=b$ be given, let 


$$
[y](s)=\sum_{j=1}^{n} \chi_{\left[t_{j-1}, t_{j}\right)}(s)\left[y\left(t_{j-1}\right)+\frac{s-t_{j-1}}{t_{j}-t_{j-1}}\left(y\left(t_{j}\right)-y\left(t_{j-1}\right)\right)\right]+y(b) \xi_{\{b\}}(s)
$$

for $y$ in $C[a, b]$ and let

$$
[u](s)=\sum_{j=1}^{n} \chi_{\left[t_{j-1}, t_{j}\right)}(s)\left[u_{j-1}+\frac{s-t_{j-1}}{t_{j}-t_{j-1}}\left(u_{j}-u_{j-1}\right)\right]+u_{n} \xi_{\{b\}}(s)
$$

for $\left(u_{0}, u_{1}, \ldots, u_{n}\right)$ in $\mathbb{R}^{n+1}$.

By [35], we have following theorem from the direct calculations $E\left(\exp \left\{i \lambda_{1} X+i \lambda_{2} Y\right\}\right)=E\left(\exp \left\{i \lambda_{1} X\right\}\right) E\left(\exp \left\{i \lambda_{2} Y\right\}\right)$ and $E\left(\exp \left\{i \lambda_{1} X+i \lambda_{3} Z\right\}\right)=E\left(\exp \left\{i \lambda_{1} X\right\}\right) E\left(\exp \left\{i \lambda_{3} Z\right\}\right)$.

Theorem 6.1. Let $\varphi$ be a probability measure on $(\mathbb{R}, \mathcal{B}(\mathbb{R}))$. Let $a=t_{0}<t_{1}<\cdots<s_{1}<t_{j-1}<s_{2}<t_{j}<s<\cdots<$ $t_{n}=b$ and let $X, Y$ and $Z$ be three functions from $C[a, b]$ into $\mathbb{R}$ with $X(y)=y(s)-[y](s), Y(y)=y\left(s_{1}\right)$ and $Z(y)=y\left(s_{2}\right)$, respectively. Then $X$ and $Y$ are stochastically independent and $X$ and $Z$ are stochastically independent.

In 2008, Professor D. H. Cho proved the next theorem by the quite different and long method on the analogue of Wiener space over paths in $\mathbb{B}[8]$, compare with our proof in [35].

Theorem 6.2 (The simple formula for conditional expectation). Let $\varphi$ be a Borel probability measure on $\mathbb{R}$. Let $J_{\vec{t}}: C[a, b] \rightarrow \mathbb{R}^{n+1}$ be the function with $J_{\vec{t}}(y)=\left(y\left(t_{0}\right), y\left(t_{1}\right), \ldots, y\left(t_{n}\right)\right)$. Let $F$ be $m_{\varphi}$-integrable on $C[a, b]$. Then for $E$ in $\mathcal{B}\left(\mathbb{R}^{n+1}\right)$,

$$
\begin{aligned}
& {\left[(B a)-\int_{C(\mathbb{B})} F(y) d V_{J_{\vec{t}}}^{\varphi}(y)\right](E)} \\
& \quad=\int_{J_{\vec{t}}^{-1}(E)} F(y) d m_{\varphi}(y) \\
& \quad=\int_{E}\left(\int_{C([a, b])} F(y-[y]+[\vec{u}]) d m_{\varphi}(y)\right) d P_{J_{\vec{t}}}^{\varphi}(\vec{u}) \\
& \quad=\int_{E} E^{\varphi}\left(F \mid J_{\vec{t}}\right) d P_{J_{\vec{t}}}^{\varphi}(\vec{u}) .
\end{aligned}
$$

That is,

$$
E^{\varphi}\left(F \mid J_{\vec{t}}\right)=E(F(y-[y]+[\vec{u}])) .
$$

We know that for any bounded measurable function $F$ on $C[a, b]$ and for any probability measure $\varphi$ on $(\mathbb{R}, \mathcal{B}(\mathbb{R}))$, there is a conditional expectation $E^{\varphi}\left(F \mid J_{\vec{t}}\right)$. What happen if the probability measure $\varphi$ change?

Theorem 6.3 (The uniqueness theorem for giving distributions). For a bounded measurable function $F$ on $C[a, b]$, there is a unique conditional expectation $E\left(F \mid J_{\vec{t}}\right)$, independent of the selection of the distribution $\varphi$ such that

$$
\left[(B a)-\int_{C[a, b]} F(x) d V_{J_{\vec{t}}}^{\varphi}(x)\right](E)=\int_{E} E\left(F \mid J_{\vec{t}}\right)(\vec{u}) d P_{J_{\vec{t}}}^{\varphi}(\vec{u})
$$

for any $E$ in $\mathcal{B}\left(\mathbb{R}^{n+1}\right)$ and for any Borel probability measure $\varphi$ on $\mathbb{R}$.

Remark 6.4. In Theorem 6.3, if we take $\varphi=\delta_{0}$ then $u_{0}$ doesn't appear in the representation of $E\left(F \mid J_{\vec{t}}\right)$ because $u_{0}=0$.

\section{A measure-valued Feynman-Kac formula}

Cameron and storvick introduced an operator-valued function space integral in 1968 [5]. Johnson and Lapidus established the existence theorem of the operator-valued function space integral as an operator from $L_{2}\left(\mathbb{R}^{N}\right)$ to itself for certain functionals involving some Borel measures [16]. And in 1987, Lapidus proved that the integral satisfies the Schrödinger wave equation [20]. In 1992, Chang and the first author established the existence theorem of the operatorvalued function space integral as an operator from $L_{p}$ to $L_{p^{\prime}}(1<p<2)$ for certain functionals involving some Borel measures [7]. The first author proved that the integral satisfies a Volterra-Stieljes integral equation in [33] In this section, we will achieve the measure-valued Feynman-Kac formula for the integral with respect to a measure-valued measure of suitable functional. Throughout in this section and the next sections, we let $X(x)=x(b)$ and $V_{\varphi}^{X}=V_{\varphi}$ [36].

Theorem 7.1. Let $\varphi$ be in $\mathcal{M}(\mathbb{R})$, let $\eta$ be a complex-valued Borel measure on $[a, b]$ and let $\theta$ be in $L_{\varphi ; \infty, 1 ; \eta}$. Then

$$
|\theta(s, x(s))| \leq\|\theta(s, \cdot)\|_{\varphi ; \infty}
$$

for $|\eta| \times \omega_{|\varphi|}$-a.e. $(s, x)$ in $[a, b] \times C[a, b]$. 
Throughout this section let $\eta=\mu+v$ be a complex-valued Borel measure on $[a, b]$ such that $\mu$ is the continuous part of $\eta$ and $v=\sum_{p=0}^{n} c_{p} \delta_{\tau_{p}}$ where $a=\tau_{0}<\tau_{1}<\tau_{2}<\cdots<\tau_{n}=b$ and $c_{p}(p=0,1, \ldots, n)$ are complex numbers, let $\varphi$ be in $\mathcal{M}(\mathbb{R})$ and let $\theta$ be in $L_{\varphi ; \infty, 1 ; \eta}$. For non-negative integers $q$ and $j_{1}, \ldots, j_{n}$ with $q=j_{1}+j_{2}+\cdots, j_{n}$, let

$$
\begin{aligned}
\triangle_{q ; j_{1}, j_{2}, \ldots, j_{n}} & \\
= & \left\{\left(s_{1,1}, s_{1,2}, \ldots, s_{1, j_{1}}, s_{2,1}, \ldots, s_{n-1, j_{n-1}}, s_{n, 1}, \ldots, s_{n, j_{n}}\right) \mid \tau_{0}=a<s_{1,1}<\right. \\
& \left.\quad \cdots<s_{1, j_{1}}<\tau_{1}<s_{2,1}<\cdots<\tau_{n-1}<s_{n, 1}<\cdots<s_{n, j_{n}}<\tau_{n}=b\right\} .
\end{aligned}
$$

For convenience, we let $M_{\theta(s,)} \equiv M_{\theta(s)}$ for $a \leq s \leq b$ and $\tau_{0}=s_{0,0}, \tau_{n}=t=s_{n, j_{n}+1}$ and $\tau_{k}=s_{k+1,0}=s_{k, j_{k}+1}$ for $k=1,2, \ldots, n-1$. For non-negative integers $m, q_{0}, \ldots, q_{n+1}, j_{1}, \ldots, j_{n}$ with $m=q_{0}+q_{1}+\cdots+q_{n+1}$ and $q_{n+1}=j_{1}+j_{2}+\cdots+j_{n}$, let $K(m, n, q, j): \triangle_{q_{n+1} ; j_{1}, j_{2}, \ldots, j_{n}} \times C[a, b] \rightarrow \mathbb{C}$ be a function with

$$
K(m, n, q, j)\left(\left(s_{1,1}, \ldots, s_{n, j_{n}}\right), x\right)=\left[\prod_{i=0}^{n} \theta\left(\tau_{i}, x\left(\tau_{i}\right)\right)^{q_{i}}\right]\left[\prod_{i=1}^{n} \prod_{j=1}^{j_{i}} \theta\left(s_{i, j}, x\left(s_{i, j}\right)\right)\right]
$$

and let $D(m, n, q, j): \triangle_{q_{n+1} ; j_{1}, j_{2}, \ldots, j_{n}} \rightarrow \mathbb{R}$ be a function with

$$
D(m, n, q, j)\left(s_{1,1}, \ldots, s_{n, j_{n}}\right)=\left[\prod_{i=0}^{n}\left\|\theta\left(\tau_{i}, \cdot\right)\right\|_{\varphi ; \infty}^{q_{i}}\right]\left[\prod_{i=1}^{n} \prod_{j=1}^{j_{i}}\left\|\theta\left(s_{i, j}, \cdot\right)\right\|_{\varphi ; \infty}\right] .
$$

Lemma 7.2. (1) $|K(m, n, q, j)| \leq D(m, n, q, j)|\mu| \times \omega_{|\varphi|}$-a.e.

(2)

$$
\begin{aligned}
& \left|\int_{\triangle_{q_{n+1} ; j_{1}, \ldots, j_{n}}} D(m, n, q, j)\left(s_{1,1}, \ldots, s_{n, j_{n}}\right) d\left(\prod_{i=1}^{n} \prod_{j=1}^{j_{i}} \mu\right)\left(s_{1,1}, \ldots, s_{n, j_{n}}\right)\right| \\
& \quad \leq \frac{1}{q_{n+1} !}\left(\prod_{i=0}^{n}\left\|\theta\left(\tau_{i}, \cdot\right)\right\|_{\varphi ; \infty}^{q_{i}}\right)\left(\|\theta\|_{\varphi ; \infty, 1 ; \mu}\right)^{q_{n+1}}
\end{aligned}
$$

(3) $D(m, n, q, j)$ is $\left(\prod_{i=1}^{n} \prod_{j=1}^{j_{i}} \mu\right) \times V_{\varphi}$-Bartle integrable on $\triangle_{q_{n+1} ; j_{1}, \ldots, j_{n}} \times C[0, t]$.

Lemma 7.3. $\theta(s, x(s))$ is $\mu \times V_{\varphi}$-Bartle integrable on $[0, t] \times C[0, t]$.

Theorem 7.4. (1) $K(m, n, q, j)$ is $\left(\prod_{i=1}^{n} \prod_{j=1}^{j_{i}} \mu\right) \times V_{\varphi}$-Bartle integrable.

(2) for $\prod_{i=1}^{n} \prod_{j=1}^{j_{i}}|\mu|$-a.e. $\left(s_{1,1}, \ldots, s_{n, j_{n}}\right), K(m, n, q, j)\left(\left(s_{1,1}, \ldots, s_{n, j_{n}}\right), \cdot\right)$ is $V_{\varphi}$-Bartle integrable.

(3) $(B a)-\int_{C[0, t]} K(m, n, q, j)\left(\left(s_{1,1}, \ldots, s_{n, j_{n}}\right), x\right) d V_{\varphi}(x)$ is $\prod_{i=1}^{n} \prod_{j=1}^{j_{i}} \mu$-Bochner integrable.

The proof of the following theorem is patterned to some extent on earlier work by Johnson and Lapidus in [9] but the present setting requires a number of new concepts and results in the previous parts of this section.

Theorem 7.5 (A measure-valued Feynman-Kac formula). $\exp \left\{\int_{[a, b]} \theta(s, x(s)) d \eta(s)\right\}$ is $V_{\varphi}$-Bartle integrable on $C[a, b]$ and for $E$ in $\mathcal{B}(\mathbb{R})$,

$$
\begin{aligned}
& {\left[(B a)-\int_{C[a, b]} \exp \left\{\int_{[a, b]} \theta(s, x(s)) d \eta(s)\right\} d V_{\varphi}(x)\right](E)} \\
& =\sum_{m=0}^{\infty} \sum_{q_{0}+\cdots+q_{n+1}=m} \frac{\prod_{p=0}^{n} c_{p}^{q_{p}}}{\prod_{p=0}^{n} q_{p} !} \sum_{j_{1}+\cdots+j_{n}=q_{n+1}} \int_{\triangle_{q_{n+1} ; j_{1}, \ldots, j_{n}}} \\
& \quad\left[\left(L_{n} \circ L_{n-1} \circ \cdots \circ L_{1}\right)\left(T\left(s_{1,1}, \varphi, \theta(0, \cdot)^{q_{0}}\right)\right)\right](E) d\left(\prod_{i=1}^{n} \prod_{j=1}^{j_{i}} \mu\right)\left(s_{1,1}, \ldots, s_{n, j_{n}}\right) .
\end{aligned}
$$

Moreover,

$$
\begin{aligned}
& \left|(B a)-\int_{C[a, b]} \exp \left\{\int_{[a, b]} \theta(s, x(s)) d \eta(s)\right\} d V_{\varphi}(x)\right|(\mathbb{R}) \\
& \quad \leq 4|\varphi|(\mathbb{R})\left[\exp \left\{\|\theta\|_{\varphi ; \infty, 1 ; \eta}\right\}\right] .
\end{aligned}
$$

Here, for $k=2,3, \ldots, n$,

$$
L_{k}=M_{\theta\left(\tau_{k}\right)^{q_{k}}} \circ S_{\tau_{k}-s_{k, j_{k}}} \circ M_{\theta\left(s_{k, j_{k}}\right)} \circ S_{s_{k, j_{k}}-s_{k, j_{k}-1}} \circ \cdots \circ M_{\theta\left(s_{k, 1}\right)} \circ S_{s_{k, 1}-s_{k, 0}}
$$

and 


$$
L_{1}=M_{\theta\left(\tau_{1}\right)^{q_{1}}} \circ S_{\tau_{1}-s_{1, j_{1}}} \circ M_{\theta\left(s_{1, j_{1}}\right)} \circ S_{s_{1, j_{1}}-s_{1, j_{1}-1}} \circ \cdots \circ M_{\theta\left(s_{1,1}\right)} .
$$

From Theorem 7.5, directly we deduce the following corollaries.

Corollary 7.6. In Theorem 7.5, we assume that $\eta=\mu$, an arbitrary continuous measure on $[a, b]$. Then for $E$ in $\mathcal{B}(\mathbb{R})$

$$
\begin{aligned}
& {\left[(B a)-\int_{C[a, b]} \exp \left\{\int_{[a, b]} \theta(s, x(s)) d \eta(s)\right\} d V_{\varphi}(x)\right](E)} \\
& =\sum_{m=0}^{\infty} \int_{\triangle_{m}}\left[\left(S_{t-s_{m}} \circ M_{\theta\left(s_{m}\right)} \circ \cdots \circ S_{s_{2}-s_{1}} \circ M_{\theta\left(s_{1}\right)}\right)\left(T\left(s_{1}, \varphi, \theta^{0} \equiv 1\right)\right)\right](E) \\
& \quad \times d\left(\prod_{i=1}^{m} \mu\right)\left(s_{1}, s_{2}, \ldots, s_{m}\right),
\end{aligned}
$$

where $\triangle_{m}=\left\{\left(s_{1}, s_{2}, \ldots, s_{m}\right)\right.$ in $\left.[0, t]^{m} \mid 0<s_{1}<s_{2}<\cdots<s_{m}<t\right\}$.

Corollary 7.7. In Theorem 7.5, we assume that $\eta=v=\sum_{p=0}^{n} c_{p} \delta_{\tau_{p}}$, a discrete measure on $[a, b]$ with finite support. Then for $E$ in $\mathcal{B}(\mathbb{R})$,

$$
\begin{aligned}
{[(B a)-} & \left.\exp \left\{\int_{C[a, b]} \theta(s, x(s)) d \eta(s)\right\} d V_{\varphi}(x)\right](E) \\
= & \sum_{m=0}^{\infty} \sum_{q_{0}+\cdots+q_{n}=m} \frac{\prod_{p=0}^{n} c_{p}^{q_{p}}}{\prod_{p=0}^{n} q_{p} !}\left[\left(M_{\theta\left(\tau_{n}\right)^{q_{n}}} \circ S_{\tau_{n}-\tau_{n-1}} \circ \cdots \circ S_{\tau_{2}-\tau_{1}} \circ M_{\theta\left(\tau_{1}\right)^{q_{1}}}\right)\right. \\
& \left.\left(T\left(\tau_{1}, \varphi, \theta(0, \cdot)^{q_{0}}\right)\right)\right](E) .
\end{aligned}
$$

Corollary 7.8. In Theorem 7.5, we assume that $c_{n}=0$. Then for $E$ in $B(\mathbb{R})$,

$$
\begin{aligned}
& {\left[(B a)-\int_{C[a, b]} \exp \left\{\int_{[a, b]} \theta(s, x(s)) d \eta(s)\right\} d V_{\varphi}(x)\right](E)} \\
& =\sum_{m=0}^{\infty} \sum_{q_{0}+\cdots+q_{n}=m} \frac{\prod_{p=0}^{n-1} c_{p}^{q_{p}}}{\prod_{p=0}^{n-1} q_{p} !} \sum_{j_{1}+\cdots+j_{n}=q_{n}} \int_{\triangle_{q_{n} ; j_{1}, \ldots, j_{n}}} \\
& {\left[\left(\left(S_{t-s_{n, j_{n}}} \circ M_{\theta\left(s_{n, j_{n}}\right)} \circ \cdots \circ S_{S_{n, 1}-\tau_{n-1}}\right) \circ L_{n-1} \circ \cdots \circ L_{1}\right)\right.} \\
& \left.\left(T\left(s_{1,1}, \varphi, \theta(0, \cdot)^{q_{0}}\right)\right)\right](E) d\left(\prod_{i=1}^{n} \prod_{j=1}^{j_{i}} \mu\right)\left(s_{1,1}, \ldots, s_{n, j_{n}}\right) .
\end{aligned}
$$

\section{A Volterra integral equation for the measure-valued Feynman-Kac formula}

In this section, we prove that the equality in Theorem 7.5, satisfies a suitable Volterra integral equation.

Throughout this section, let $a=0=\tau_{0}<\tau_{1}<\cdots<\tau_{n}=t<\tilde{t}=b$ and let $\eta$ be a Borel measure on $[0, \tilde{t}]$ such that $\eta=\mu+v$ where $\mu$ is the continuous part of $\eta$ and $v=\sum_{p=0}^{n} c_{p} \delta_{\tau_{p}}$; further let $\theta$ be in $L_{\varphi ; \infty, 1 ; \eta}^{\tilde{t}}$. Let

$$
u\left(t^{\prime}\right)=(B a)-\int_{C\left[0, t^{\prime}\right]} \exp \left\{\int_{\left[0, t^{\prime}\right]} \theta(s, x(s)) d \eta(s)\right\} d V_{\varphi}(x)
$$

for $t<t^{\prime} \leq \tilde{t}$.

The following theorem is the counterpart for the measure-valued measure $V_{\varphi}$ of the integral equation for the Feynman-Kac formula with Lebesgue-Stieljes measure, obtained by Lapidus in [20-22] and for the Feynman-Kac formula with an operator-valued measure, obtained by Kluvanek in [18].

Theorem 8.1 (The measure-valued Feynman-Kac formula). For $t<t^{\prime} \leq \widetilde{t}, u\left(t^{\prime}\right)$ satisfies a Volterra integral equation, that is,

$$
u\left(t^{\prime}\right)=S_{t^{\prime}-t}(u(t))+(B o)-\int_{\left(t, t^{\prime}\right]}\left(S_{t^{\prime}-s} \circ M_{\theta(s)}\right) u(s) d \mu(s)
$$

Corollary 8.2. Under the assumptions in Corollary 7.6, for $0<t^{\prime} \leq \widetilde{t}, u\left(t^{\prime}\right)$ satisfies a Volterra integral equation, that is, 


$$
u\left(t^{\prime}\right)=S_{t^{\prime}}(\varphi)+(B o)-\int_{\left(0, t^{\prime}\right]}\left(S_{t^{\prime}-s} \circ M_{\theta(s)}\right)(u(s)) d \mu(s)
$$

Corollary 8.3. Under the assumptions in Corollary 7.7, for $0<t^{\prime} \leq \tilde{t}$,

$$
\begin{aligned}
u\left(t^{\prime}\right) & =\sum_{m=0}^{\infty} \sum_{q_{0}+\cdots+q_{n}=m} \frac{\prod_{p=0}^{n} c_{p}^{q_{p}}}{\prod_{p=0}^{n} q_{p} !}\left[S_{t^{\prime}-t} \circ M_{\theta\left(\tau_{n}\right)^{q_{n}}} \circ S_{\tau_{n}-\tau_{n-1}} \circ \cdots \circ S_{\tau_{2}-\tau_{1}} \circ M_{\theta\left(\tau_{1}\right)^{q_{1}}}\right] \\
& \left(T\left(\tau_{1}, \varphi, \theta(0, \cdot)^{q_{0}}\right)\right),
\end{aligned}
$$

$$
u\left(t^{\prime}\right)=S_{t^{\prime}-t}(u(t))
$$

and

$$
\left.(B o)-\int_{\left(t, t^{\prime}\right]}\left(S_{t^{\prime}-s} \circ M_{\theta(s)}\right)(u(s)) d \mu(s)=0 \quad \text { (the zero operator }\right) .
$$

\section{The Dobrakov integral on the analogue of Wiener space}

In this section, we will treat the theory of Dobrakov integral over $C[a, b]$. For $B$ in $\mathcal{B}(C[a, b])$, letting $V(B)$ : $\mathcal{M}(\mathbb{R}) \rightarrow \mathcal{M}(\mathbb{R})$ with $[V(B)](\varphi)=V_{\varphi}(B), V(B)$ is a bounded linear operator on $\mathcal{M}(\mathbb{R})$. From [34], we can check that the following facts.

Lemma 9.1. For $u_{0}$ in $\mathbb{R}$, let $P_{u_{0}}=\left\{x \in C[a, b] \mid x(a)=u_{0}\right\}$. Then $\hat{V}\left(P_{u_{0}}\right)=1$.

Theorem 9.2. Let $F$ be a subset of $\mathbb{R}$ and let $P(F)=\{x \in C[a, b] \mid x(0)$ belongs to $F\}$. Then $F$ is finite if and only if $\hat{V}(P(F))$ is finite if and only if $P(F)$ is integrable.

Using the Theorem 2.4, we have the following theorem.

Theorem 9.3. $V$ is an operator-valued measure countably additive in the strong operator topology but is not an operator-valued measure countably additive in the uniform operator topology.

Theorem 9.4. $\mathcal{M}(\mathbb{R})$ is not a weakly complete Banach space i.e., there is a subspace of $\mathcal{M}(\mathbb{R})$ which is isomorphic to the space $c_{0}$.

Theorem 9.5. If $F$ is a finite subset of $\mathbb{R}$, then $V$ is continuous on $P_{0}(F)$.

Lemma 9.6. Let the semivariation $\hat{m}$ be continuous on an integrable set, let $A$ be an integrable set and let $f$ be a bounded strongly function. Then the function $f \cdot \chi_{A}$ integrable.

Theorem 9.7 (The Wiener integral formula for operator-valued measure). Let $F=\left\{u_{1}, u_{2}, \ldots, u_{n}\right\}$ and let $a=$ $t_{0}<t_{1}<\cdots<t_{n}=b$ be given. Suppose $H$ is a function from $P(F)$ into $\mathcal{M}(\mathbb{R})$ such that $H(x)=\delta_{x(a)} h_{x(a)}$ $\left(x\left(t_{1}\right), x\left(t_{2}\right), \ldots, x\left(t_{n}\right)\right)$ and $\left.h_{u_{k}} k=1,2, \ldots, n\right)$ are bounded measurable functions on $\mathbb{R}^{n}$. Then the Dobrakov integral $\int_{P(F)} H(x) d V(x)$ exists and the following equality holds.

$$
\begin{aligned}
& {\left[\int_{P(F)} H(x) d V(x)\right](E)} \\
& \quad=\frac{1}{\prod_{i=1}^{n} \sqrt{2 \pi\left(t_{i}-t_{i-1}\right)}} \sum_{k=1}^{n} \int_{E}\left[\int_{\mathbb{R}^{n-1}} h_{u_{k}}\left(v_{1}, v_{2}, \ldots, v_{n}\right)\right. \\
& \quad \exp \left\{-\frac{1}{2} \sum_{i=2}^{n} \frac{\left(v_{i}-v_{i-1}\right)^{2}}{t_{i}-t_{i-1}}\right\} \exp \left\{-\frac{1}{2} \frac{\left(v_{1}-u_{k}\right)^{2}}{t_{1}}\right\} \\
& \left.\quad d m_{L}\left(v_{1}\right) \cdots d m_{L}\left(v_{n-1}\right)\right] d m_{L}\left(v_{n}\right)
\end{aligned}
$$

for all Borel subset $E$ of $\mathbb{R}$.

Example 9.8. Let $t=2$ and let $F=\{3,5\}$. Let $H(x)=\delta_{x(0)} \exp \left\{-x(1)^{2}\right\}$. Then for $E \in \mathscr{B}(\mathbb{R})$, 


$$
\begin{aligned}
& {\left[\int_{P(E)} H(x) d V(X)\right](E)} \\
& \quad=\int_{E}\left[\int_{\mathbb{R}} \frac{1}{\sqrt{(2 \pi)^{2}}} \exp \left\{-u_{1}^{2}-\frac{1}{2}\left(u_{2}-u_{1}\right)^{2}-\frac{1}{2}\left(u_{1}-3\right)^{2}\right\} d m_{L}\left(u_{1}\right)\right] d m_{L}\left(u_{2}\right) \\
& \quad+\int_{E}\left[\int_{\mathbb{R}} \frac{1}{\sqrt{(2 \pi)^{2}}} \exp \left\{-u_{1}^{2}-\frac{1}{2}\left(u_{2}-u_{1}\right)^{2}-\frac{1}{2}\left(u_{1}-5\right)^{2}\right\} d m_{L}\left(u_{1}\right)\right] d m_{L}\left(u_{2}\right) \\
& =\frac{e^{-3}}{2 \sqrt{2 \pi}} \int_{E} \exp \left\{-\frac{3}{8}\left(u_{2}-1\right)^{2}\right\} d u_{2}+\frac{e^{-\frac{25}{3}}}{2 \sqrt{2 \pi}} \int_{E} \exp \left\{-\frac{3}{8}\left(u_{2}-\frac{5}{3}\right)^{2}\right\} d u_{2} .
\end{aligned}
$$

\section{The operational calculus for a measure-valued Dyson series}

In this section, we investigate Feynman's operational calculus for a measure-valued Dyson series [32]. Throughout this section, let $t_{1}$ and $t_{2}$ be two real numbers with $0<t_{1}<t_{2}$ and let $\varphi$ be in $\mathcal{M}(\mathbb{R})$. Let $X^{a, b}: C[a, b] \rightarrow \mathbb{R}$ be a function with $X^{a, b}(y)=y(b)$ for $a<b$. For $E$ in $\mathscr{B}(\mathbb{R})$ and for $B$ in $\mathcal{B}(C[a, b]), V_{\varphi}^{a, b}(B)=\omega_{\varphi}\left(B \cap X^{a, b}(E)\right)$.

Theorem 10.1. Let $\left(s_{0}, s_{1}, s_{2}, \ldots, s_{m+n}\right)$ be in $\mathbb{R}^{m+n+1}$ with $0<s_{0}<s_{1}<\cdots<s_{m}=t_{1}<s_{m+1}<\cdots<s_{m+n}=t_{2}$. Let $f_{1}$ and $f_{2}$ be two complex-valued Borel measurable functions on $\mathbb{R}^{m+1}$ and $\mathbb{R}^{n}$, respectively such that

$$
f_{1}\left(u_{0}, u_{1}, \ldots, u_{m}\right) W\left(m+1 ;\left(s_{0}, s_{1}, \ldots, s_{m}\right) ; u_{0}, u_{1}, \ldots, u_{m}\right)
$$

is $|\varphi| \times \prod_{j=1}^{m} m_{L}$-integrable on $\mathbb{R}^{m+1}$ and

$$
\begin{aligned}
& f_{1}\left(u_{0}, u_{1}, \ldots, u_{m}\right) f_{2}\left(u_{m+1}, \ldots, u_{m+n}\right) \\
& \quad \times W\left(m+n+1 ;\left(s_{0}, s_{1}, \ldots, s_{m+n+1}\right) ; u_{0}, u_{1}, \ldots, u_{m+n}\right)
\end{aligned}
$$

is $|\varphi| \times \prod_{j=1}^{m+n} m_{L}$-integrable on $\mathbb{R}^{m+n+1}$. Then

$$
F_{1}(x)=f_{1}\left(x\left(s_{0}\right), x\left(s_{1}\right), \ldots, x\left(s_{m}\right)\right)
$$

is $V_{\varphi}^{0, t_{1}}$-Bartle integrable on $C\left[0, t_{1}\right]$,

$$
F(x)=f_{1}\left(x\left(s_{0}\right), x\left(s_{1}\right), \ldots, x\left(s_{m}\right)\right) f_{2}\left(x\left(s_{m+1}\right), \ldots, x\left(s_{m+n}\right)\right)
$$

is $V_{\varphi}^{0, t_{2}}$-Bartle integrable on $C\left[0, t_{2}\right]$ and

$$
F_{2}(x)=f_{2}\left(x\left(s_{m+1}\right), \ldots, x\left(s_{m+n}\right)\right)
$$

is $V_{\tilde{\varphi}}^{t_{1}, t_{2}}$-Bartle integrable on $C\left[t_{1}, t_{2}\right]$, where

$$
\tilde{\varphi}(E)=\left[(B a)-\int_{C\left[0, t_{1}\right]} F_{1}(x) d V_{\varphi}^{0, t_{1}}(x)\right](E)
$$

for $E$ in $\mathcal{B}(\mathbb{R})$. Moreover,

$$
(B a)-\int_{C\left[0, t_{2}\right]} F(x) d V_{\varphi}^{0, t_{2}}(x)=(B a)-\int_{C\left[t_{1}, t_{2}\right]} F_{2}(x) d V_{\varphi}^{t_{1}, t_{2}}(x) .
$$

Remark 10.2. Let $\varphi$ be in $\mathcal{M}(\mathbb{R})$, let $P_{1}: C\left[0, t_{2}\right] \rightarrow C\left[0, t_{1}\right]$ be a function with $\left[P_{1}(x)\right](s)=x(s)$ for $0 \leq s \leq t_{1}$ and let $P_{2}: C\left[0, t_{2}\right] \rightarrow C\left[t_{1}, t_{2}\right]$ be a function with $\left[P_{2}(x)\right](s)=x(s)$ for $t_{1} \leq s \leq t_{2}$. Then by Theorem 10.1, $V_{\varphi}^{0, t_{2}}(I)=V_{\tilde{\varphi}}^{t_{1}, t_{2}}\left(P_{2}(I)\right)$ for $I$ in $\ell$ where $\tilde{\varphi}=V_{\varphi}^{0, t_{1}}\left(P_{1}(I)\right)$. But it is not true always that $V_{\varphi}^{0, t_{2}}(B)=V_{\tilde{\varphi}}^{t_{1}, t_{2}}\left(P_{2}(B)\right)$ for $B$ in $\mathcal{B}\left(C\left[0, t_{2}\right]\right)$ where $\tilde{\varphi}=V_{\varphi}^{0, t_{1}}\left(P_{1}(B)\right)$. Because, putting $\varphi=\delta_{0}, t_{1}=1, t_{2}=2$ and $B=\left\{x \in C\left[0, t_{2}\right] \mid\right.$ either $x(1) \geq 0$ and $x(2) \geq 0$ or $x(1)<0$ and $x(2)<0$ holds $\}, B$ in $\mathcal{B}(C[0,2]),\left[V_{\varphi}^{0,2}(B)\right](\mathbb{R})=\frac{1}{2}, V_{\varphi}^{0,1}\left(P_{1}(B)\right)=V_{\varphi}^{0,1}(C[0,1])=S_{1}\left(\delta_{0}\right)$, the standard normal distribution, and

$$
\left[V_{S_{1}\left(\delta_{0}\right)}^{1,2}\left(P_{2}(B)\right)\right](\mathbb{R})=\left[S_{1} \circ S_{1}\left(\delta_{0}\right)\right](\mathbb{R})=\left[S_{2}\left(\delta_{0}\right)\right](\mathbb{R})=1 .
$$

Here, we establish the operational calculus for a measure-valued Dyson series, the following theorem in this note.

Theorem 10.3. Under the assumptions in the above theorem, let $g(z)=\exp (z)$ and let $\eta=\mu+v$ be a complexvalued Borel measure on $\left[0, t_{2}\right]$ such that $\mu$ is a continuous part of $\eta$ and $\nu=\sum_{p=0}^{m+n} c_{p} \delta_{\tau_{p}}$ where $0=\tau_{0}<\tau_{1}<\cdots<$ $\tau_{m}=t_{1}<\tau_{m+1}<\cdots<\tau_{m+n}=t_{2}$ and $c_{p}(p=0,1, \ldots, m+n)$ are complex numbers. Then $\exp \left(\int_{\left[0, t_{1}\right]} \theta(s, x(s)) d \eta(s)\right)$, $\exp \left(\int_{\left(t_{1}, t_{2}\right]} \theta(s, x(s)) d \eta(s)\right)$ and $\exp \left(\int_{\left[0, t_{2}\right]} \theta(s, x(s)) d \eta(s)\right)$ are all $V_{\varphi}^{0, t_{1}}$, $V_{\tilde{\varphi}}^{t_{1}, t_{2}}$ - and $V_{\varphi}^{0, t_{2}}$-Bartle integrable of $x$ on $C\left[0, t_{1}\right]$, $C\left[t_{1}, t_{2}\right]$ and $C\left[0, t_{2}\right]$, respectively, where 


$$
\tilde{\varphi}=(B a)-\int_{C\left[0, t_{1}\right]} \exp \left(\int_{\left[0, t_{1}\right]} \theta(s, x(s)) d \eta(s)\right) d V_{\varphi}^{0, t_{1}}(x) .
$$

Moreover,

$$
\begin{aligned}
& (B a)-\int_{C\left[0, t_{2}\right]} \exp \left(\int_{\left[0, t_{2}\right]} \theta(s, x(s)) d \eta(s)\right) d V_{\varphi}^{0, t_{2}}(x) \\
& =(B a)-\int_{C\left[t_{1}, t_{2}\right]} \exp \left(\int_{\left(t_{1}, t_{2}\right]} \theta(s, x(s)) d \eta(s)\right) d V_{\tilde{\varphi}}^{t_{1}, t_{2}}(x) .
\end{aligned}
$$

Remark 10.4. (a) Let $\theta$ be a constant function on $\left[0, t_{2}\right] \times \mathbb{R}$, say $\theta(s, u)=c$, let $\eta$ be the Lebesgue measure on $\left[0, t_{2}\right]$ and let $\varphi=\delta_{0}$. Then

$$
\int_{C\left[0, t_{1}\right]} \exp \left(\int_{\left[0, t_{1}\right]} \theta(s, x(s)) d \eta(s)\right) d V_{\varphi}^{0, t_{1}}(x)=\exp \left(c t_{1}\right) S_{t_{1}}\left(\delta_{0}\right) \equiv \tilde{\varphi}
$$

and

$$
\begin{aligned}
& \int_{C\left[t_{1}, t_{2}\right]} \exp \left\{\int_{\left[t_{1}, t_{2}\right]} \theta(s, x(s)) d \eta(s)\right\} d V_{\tilde{\varphi}}^{t_{1}, t_{2}}(x) \\
& =\exp \left(c\left(t_{2}-t_{1}\right)\right) S_{t_{2}-t_{1}}(\tilde{\varphi}) \\
& =\exp \left(c t_{2}\right) S_{t_{2}}\left(\delta_{0}\right) \\
& =\int_{C\left[0, t_{2}\right]} \exp \left(\int_{\left[0, t_{2}\right]} \theta(s, x(s)) d \eta(s)\right) d V_{\varphi}^{0, t_{2}}(x),
\end{aligned}
$$

so, a formula, given in Theorem 10.3 , holds.

(b) Taking $g(z)=z$ in (a)

$$
\int_{C\left[0, t_{1}\right]} g\left(\int_{\left[0, t_{1}\right]} \theta(s, x(s)) d \eta(s)\right) d V_{\varphi}^{0, t_{1}}(x)=c t_{1} S_{t_{1}}\left(\delta_{0}\right) \equiv \psi
$$

and

$$
\begin{aligned}
& \int_{C\left[t_{1}, t_{2}\right]} g\left(\int_{\left[t_{1}, t_{2}\right]} \theta(s, x(s)) d \eta(s)\right) d V_{\psi}^{t_{1}, t_{2}}(x) \\
& =c\left(t_{2}-t_{1}\right) S_{t_{2}-t_{1}}(\psi)=c^{2} t_{1}\left(t_{2}-t_{1}\right) S_{t_{2}}\left(\delta_{0}\right) .
\end{aligned}
$$

Since

$$
\int_{C\left[0, t_{2}\right]} g\left(\int_{\left[0, t_{2}\right]} \theta(s, x(s)) d \eta(s)\right) d V_{\varphi}^{0, t_{2}}(x)=c t_{2} S_{t_{2}}\left(\delta_{0}\right),
$$

the formula, given in Theorem 10.3, doesn't hold for the general function $g$.

\section{Fourier-Feynman transform on analogue of Wiener space}

In this section, we will develop the theories of fourier-Feynman transform on analogue of Wiener space. First of all, we will establish the existence theorem for our transform. Moreover, we will find the properties of it. In developing our theories, the rotation theorem is key role, so we assume that a measure $\varphi$ has the Radon-Nikodym derivative with respect to the Lebesgue measure having a form $\frac{d \varphi}{d m_{L}}(x)=A e^{-a x^{2}}$ where $A, \alpha$ are two positive real numbers.

Lemma 11.1. For a non-zero complex number $\lambda$ with $\operatorname{Re} \lambda \geq 0$ and for $f \in L_{2}\left(\mathbb{R}^{n+1}\right)$, define

$$
\begin{aligned}
& g\left(v_{0}, v_{1}, \ldots, v_{n}\right) \\
& =\left(\frac{\lambda}{2 \pi}\right)^{\frac{n+1}{2}} \int_{\mathbb{R}} \cdots \int_{\mathbb{R}} f\left(u_{0}, u_{1}, \ldots, u_{n}\right) e^{-\frac{\lambda}{2} \sum_{j=0}^{n}\left(u_{j}-v_{j}\right)^{2}} d m_{L}\left(u_{0}\right) \cdots d m_{L}\left(u_{n}\right),
\end{aligned}
$$

then $g \in L_{2}\left(\mathbb{R}^{n+1}\right)$ and $\|g\|_{2} \leq\|f\|_{2}$.

Lemma 11.2. For $1 \geq p \geq 2$ and all non-zero real number $q$, if $F_{1} \stackrel{a n}{\approx} F_{2}$, then the existence of $T_{q}^{(p)}\left(F_{1}\right)$ assure the existence of $T_{q}^{(p)}\left(F_{2}\right)$, and $T_{q}^{(p)}\left(F_{1}\right) \stackrel{a n}{\approx} T_{q}^{(p)}\left(F_{2}\right)$.

Theorem 11.3. For a partition $a=t_{0}<t_{1}<\cdots<t_{n}=b$, let $F(x)=f\left(x\left(t_{0}\right), x\left(t_{1}\right), \ldots, x\left(t_{n}\right)\right)$ be in $\mathcal{A}_{n}$. Then for each $y$ in $C[a, b]$ and for all complex numbers $\lambda$ with $\operatorname{Re} \lambda>0, \int_{C[a, b]}^{a n} F(x+y) d \omega_{\varphi}(x)$ exists. Moreover, 


$$
\begin{aligned}
& \int_{C[a, b]}^{a n \text { anw } \lambda} F(x+y) d \omega_{\varphi}(x) \\
& =A \lambda^{\frac{n+1}{2}}\left[\prod_{j=1}^{n} 2 \pi\left(t_{j}-t_{j-1}\right)\right]^{-\frac{1}{2}} \int_{-\infty}^{\infty} \cdots \int_{-\infty}^{\infty} f\left(v_{0}, v_{1}, \ldots, v_{n}\right) \\
& \quad e^{-\frac{\lambda}{2} \sum_{j=1}^{n} \frac{\left[\left(v_{j}-v_{j-1}\right)-\left(y\left(t_{j}\right)-y\left(t_{j-1}\right)\right)\right]^{2}}{t_{j}-t_{j-1}}} e^{-a \lambda\left(v_{0}-y\left(t_{0}\right)\right)^{2}} \\
& \quad d m_{L}\left(v_{n}\right) \cdots d m_{L}\left(v_{1}\right) d m_{L}\left(v_{0}\right) .
\end{aligned}
$$

Moreover, let $h\left(y\left(t_{0}\right), y\left(t_{1}\right), \ldots, y\left(t_{n}\right) ; \lambda\right)$ be the right side in $(11.1)$, then

$$
\left\|h\left(y\left(t_{0}\right), y\left(t_{1}\right), \ldots, y\left(t_{n}\right) ; \lambda\right)\right\|_{2} \leq A \sqrt{\frac{\pi}{a}}\|f\|_{2}
$$

and $h\left(w_{0}, w_{1}, \ldots, w_{n} ; \lambda\right)$ is analytic of $\lambda$.

Theorem 11.4. Let $f \in L_{2}\left(\mathbb{R}^{n+1}\right)$ and let $q$ be a non-zero real number. For a partition $a=t_{0}<t_{1}<\cdots<t_{n}=b$, define

$$
\begin{aligned}
g\left(v_{0}, v_{1}, \ldots, v_{n}\right) & \\
= & \frac{A}{\sqrt{2 \pi}}(-i q)^{\frac{n+1}{2}}\left[\prod_{j=1}^{n} 2 \pi\left(t_{j}-t_{j-1}\right)\right]^{-\frac{1}{2}} \int_{-\infty}^{\infty} \cdots \int_{-\infty}^{\infty} f\left(u_{0}, u_{1}, \ldots, u_{n}\right) \\
& e^{\frac{i q}{2} \sum_{j=1}^{n} \frac{\left[\left(u_{j}-u_{j-1}\right)-\left(v_{j}-v_{j-1}\right)\right]^{2}}{t_{j}-t_{j-1}}} e^{i q a\left(u_{0}-v_{0}\right)^{2}} d m_{L}\left(u_{n}\right) \cdots d m_{L}\left(u_{1}\right) d m_{L}\left(u_{0}\right) .
\end{aligned}
$$

Then $g$ is in $L_{2}\left(\mathbb{R}^{n+1}\right)$, and for all $u_{0}, u_{1}, \ldots, u_{n}$

$$
\begin{aligned}
f\left(u_{0}, u_{1}, \ldots, u_{n}\right) & \\
= & \frac{\sqrt{2} a}{A \sqrt{\pi}}(i q)^{\frac{n+1}{2}}\left[\prod_{j=1}^{n} 2 \pi\left(t_{j}-t_{j-1}\right)\right]^{-\frac{1}{2}} \int_{-\infty}^{\infty} \cdots \int_{-\infty}^{\infty} g\left(v_{0}, v_{1}, \ldots, v_{n}\right) \\
& e^{-\frac{i q}{2} \sum_{j=1}^{n} \frac{\left[\left(u_{j}-u_{j-1}\right)-\left(v_{j}-v_{j-1}\right)\right]^{2}}{t_{j}-t_{j-1}}} e^{-i q a\left(u_{0}-v_{0}\right)^{2}} d m_{L}\left(v_{n}\right) \cdots d m_{L}\left(v_{1}\right) d m_{L}\left(v_{0}\right) .
\end{aligned}
$$

And

$$
\|f\|_{2}=\frac{4 a^{2}}{A}\|g\|_{2}
$$

Theorem 11.5. For a nonzero real number $q$ and a partition $a=t_{0}<t_{1}<\cdots<t_{n}=b$, if $F(x)=f\left(x\left(t_{0}\right), \ldots, x\left(t_{n}\right)\right)$ is in $\mathcal{A}_{n}$. Then $G \equiv T_{q}(F)$ exists and $G(y) \approx g\left(y\left(t_{0}\right), \ldots, y\left(t_{n}\right)\right) \in \mathcal{A}_{n}$ where

$$
\begin{aligned}
g\left(w_{0}, w_{1}, \ldots, w_{n}\right) & \\
= & \frac{A}{\sqrt{2 \pi}}(-i q)^{\frac{n+1}{2}}\left[\prod_{j=1}^{n} 2 \pi\left(t_{j}-t_{j-1}\right)\right]^{-\frac{1}{2}} \lim _{B \rightarrow \infty} \int_{D_{B}} \ldots \int_{D_{B}} f\left(v_{0}, v_{1}, \ldots, v_{n}\right) \\
& e^{\frac{i q}{2} \sum_{j=1}^{n} \frac{\left[\left(v_{j}-v_{j-1}\right)-\left(w_{j}-w_{j-1}\right)\right]^{2}}{t_{j}-t_{j-1}}} e^{i q a\left(v_{0}-w_{0}\right)^{2}} d m_{L}\left(v_{n}\right) \cdots d m_{L}\left(v_{1}\right) d m_{L}\left(v_{0}\right) .
\end{aligned}
$$

Moreover,

$$
\left\|T_{q}(F)\right\|_{2} \leq 4 \sqrt{a^{3}}\|g\|_{2} .
$$

\section{Acknowledgements}

This work was supported by the Korea-Japan Basic Scientific Cooperation Program (2007-2009) "Noncommutative Stochastic Analysis and Its Applications to Network Science." The authors would like to express their sincere gratitude to referee for a number of valuable comments. 


\section{REFERENCES}

[1] Bearman, J. E., "Rotation in the product of two Wiener spaces," Proc. Amer. Math. Soc., 3: 129-137 (1952).

[2] Billingsley, P., Convergence of probability measures, John Wiley \& Sons, New York (1968).

[3] Burrill, C. W., Measure, integration and probability, McGraw-Hill, New York (1972).

[4] Cameron, R. H., and Martin, W. T., "Transformations of Wiener integrals under translations," Ann. Math., 45: 386-396 (1944).

[5] Cameron, R. H., and Storvick, D. A., "An operator-valued function space integral and a related integral equation," J. Math. Mech., 18: 517-552 (1968).

[6] Cameron, R. H., and Storvick, D. A., Some Banach algebras of analytic Feynman integrable functionals, Lecture Notes 798, Springer-Verlarg (1980), 18-67.

[7] Chang, K. S., and Ryu, K. S., "Analytic operator-valued function space integrals as an $\mathcal{L}\left(L_{p}, L_{p^{\prime}}\right)$ theory," Trans. Amer. Math. Soc., 330: 697-709 (1992).

[8] Cho, D. H., "A simple formula for an analogue of conditional Wiener integrals and its applications," Trans. Amer. Math. Soc., 360: 3795-3811 (2008).

[9] Cohn, D. L., Measure theory, Birkhauser, Boston (1980).

[10] Diestel, J., and Uhl, J. J., Vector measures, Mathematical Survey, No. 15, A. M. S. (1977).

[11] Dobrakov, I., “On integration in BAnach space, I," Czech. Math. L., 20: 511-536 (1970).

[12] Dunford, N., and Schwartz, J. T., Linear operators, part I, General theory, Pure and Applied Mathematics, Vol. VII, Wiley Interscience, New York (1958).

[13] Halmos, P. R., Measure Theory, Springer-Verlag, New York (1950).

[14] Hewitt, E., and Stromberg, K., Real and Abstract Analysis, Springer-Verlag, New York (1965).

[15] Im, M. K., "The transformation theorem on analogue of Wiener space," J. Kore Soc. Math. Educ. Ser. B: Pure Appl. Math., 14(4): 317-333 (2007).

[16] Johnson, G. W., and Lapidus, M. L., "Generalized Dyson series, generalized Feynman diagrams, the Feynman integral and Feynman's operational calculus," Memoirs A. M. S., 62(351): 1-78 (1986).

[17] Johnson, G. W., and Lapidus, M. L., The Feynman integral and Feynman's operational calculus, Oxford Mathematical Monographs, Oxford Univ. Press (2000).

[18] Kluvanek, I., "Operator valued measures and Perturbations of Semi-groups," Arch. Rational Mech. Anal., 81-2: 161-180 (1983).

[19] Kluvanek, I., and Knowles, G., Vector measures and control systems, Math. Studies, No. 20, Amsterdam, North-Holland (1975).

[20] Lapidus, M. L., "The Feynman-Kac formula with a Lebesgue-Stieltjes measure and Feynman's operational calculus," Stud. Appl. Math., 76: 93-132 (1987).

[21] Lapidus, M. L., Strong product integration of measures and the Feynman-Kac formula with a Lebesgue-Stieltjes measure, Circ. Math. Palermo (2) Suppl. 17 (1987), 271-312.

[22] Lapidus, M. L., "The Feynman-Kac formula with a Lebesgue-Stieltjes measure: an integral equation in the general case," Integral Equations and Operator Theory, 12: 163-210 (1989).

[23] Larsen, R., Functional analysis, Marcel Dekker, INC, New York (1973).

[24] Lewis, D. R., "Integration with respect to vector measure," Pacific J. Math., 33(1): 157-165 (1970).

[25] Novinger, W. P., "Mean convergence in $L^{p}$ space," Proc. Amer. Math. Soc., 34: 627-628 (1972).

[26] Okikiolu, G. G., Aspect of the theory of bounded linear operators in $L_{p}$ space, Academic Press, London (1971).

[27] Parthasarathy, K. R., Probability measures on metric spaces, Academic Press, New York (1967).

[28] Reed, M., and Simon, B., Functional analysis I, Academic Press, New York (1972).

[29] Rudin, W., Real and complex analysis, 3rd. ed., Mcgraw-Hill, New York (1987).

[30] Ryu, K. S., and Im, M. K., "An analogue of Wiener measure and its applications," J. Korean Math. Soc., 39: $801-819$ (2002).

[31] Ryu, K. S., and Im, M. K., “The measure-valued Dyson series and its stability theorem,” J. Korean Math. Soc., 43: 461-489 (2006).

[32] Ryu, K. S., "The operational calculus for a measure-valued Dyson series," J. Korean Math. Soc., 43: $703-715$ (2006).

[33] Ryu, K. S., "Volterra-Stieltjes integral equation and the operator-valued function space integral as an $\mathcal{L}\left(L_{p}, L_{p^{\prime}}\right)$ theory," Bull. Korean Math. Soc., 36: 171-181 (1999).

[34] Ryu, K. S., “The Dobrakov integral over paths,” J. Chungcheong Math. Soc., 19: 61-68 (2006).

[35] Ryu, K. S., "The simple formula of conditional expectation on analogue of Wiener measure," Honam Math. J., 30: 723-732 (2008).

[36] Ryu, K. S., and Im, M. K., "A measure-valued analogue of Wiener measure and the measure-valued Feynman-Kac formula," Trans. Amer. Math. Soc., 354: 4921-4951 (2002).

[37] Ryu, K. S., and Shim, S. H., “The rotation theorem on analogue of Wiener measure," Honam Math. J., 29: 577-588 (2007).

[38] Wiener, N., "Differential space," J. Math. Phys., 2: 131-174 (1923).

[39] Yamasaki, Y., Measures on infinite dimensional space, World Scientific in Pure Math., 15 (1985).

[40] Yeh, J., Inversion of conditional expectations, Pacific J. Math., 52, No. 2 (1974), 631-640.

[41] Yeh, J., Stochastic processes and the Wiener integral, Marcel Deckker, New York (1973).

[42] Yosida, K., Functional Analysis, 4th Edition, Springer-Verlag Berlin (1974).

[43] Yoo, I., "Convolution and the Fourier-Wiener transform on abstract Wiener space," Rocky Mountain J. Math., 25: 1577-1587 (1995). 